\title{
Nonlinear energy transfer between fluid sloshing and vessel motion
}

\author{
By M. R. TURNER AND T. J. BRIDGES \\ Department of Mathematics, University of Surrey, Guildford, Surrey GU2 7XH, U.K.
}

(Received 2 January 2013)

This paper examines the dynamic coupling between a sloshing fluid and the motion of the vessel containing the fluid. A mechanism is identified which leads to an energy exchange between the vessel dynamics and fluid motion. It is based on a 1:1 resonance in the linearized equations, but nonlinearity is essential for the energy transfer. For definiteness, the theory is developed for Cooker's pendulous sloshing experiment. The vessel has a rectangular cross section, is partially filled with a fluid, and is suspended by two cables. A nonlinear normal form is derived close to an internal 1:1 resonance, with the energy transfer manifested by a heteroclinic connection which connects the purely symmetric sloshing modes to the purely anti-symmetric sloshing modes. Parameter values where this pure energy transfer occurs are identified. In practice, this energy transfer can lead to sloshing-induced destabilization of fluid-carrying vessels.

\section{Introduction}

A moving vessel containing liquid can give rise to a wide range of complex and beautiful fluid motions. On the other hand, the movement of the interior fluid induces forces and moments on the vessel that can then lead to unintended vessel motion and even a destabilization of the vessel dynamics. An everyday example is 'walking with coffee' (Mayer \& Krechetnikov 2012). A dramatic example with serious consequences is the sloshing-induced destabilization of Alaskan king crab boats (cf. Caglayan \& Storch (1982); Adee \& Caglayan (1982); Dillingham (1981)). They are very sensitive to the slosh-enhanced roll motion, and capsize can occur: trapped water on deck sloshes back and forth and creates moments which enhance roll motion (Caglayan \& Storch 1982).

The problem of fluid sloshing in stationary or forced vessels is already a very difficult one to study theoretically or experimentally as is highlighted in the works of Ibrahim (2005) and Faltinsen \& Timokha (2009). The coupled dynamics between fluid sloshing and vessel motion brings in a new dimension and the potential for enhancing or diminishing the sloshing motion through vehicle dynamics. Understanding dynamic coupling is of great practical interest in areas such as terrestrial fluid transportation, maritime and space transport, storage tanks under earthquake excitation, and industrial applications.

The interest in this paper is in a more subtle impact of sloshing on vehicle dynamics: the direct transfer of energy from fluid motion to vehicle motion through resonance and nonlinearity. The form of the energy transfer of interest is the following. Given a dynamically-coupled system, choose initial conditions so that fluid sloshing occurs but with the vessel stationary. This motion may be unstable and able to transfer all its energy to a mode in which the vessel is in motion. This type of energy transfer is essentially nonlinear, but is induced by a resonance in the linearized coupled system. In this paper a theory and its implications are developed for this nonlinear energy transfer mechanism between sloshing and vessel motion are developed.

General aspects of the dynamic coupling between vessel motion and interior fluid motion are discussed in the book of Moiseyev \& Rumyantsev (1968). They start with the fully coupled three-dimensional problem with six degree-of-freedom vessel motion. They also look at a simplified pendulum-based on model (see e.g. \$5.4 of Moiseyev \& Rumyantsev (1968)). Their model involves a single pendulum containing fluid. However this configuration would be difficult to construct experimentally, it is not natural for a shallow water configuration, and its resonance structure is quite different (the natural resonance would be a 1:2 resonance). On the other hand Cooker (1994) devised a novel coupled system which has the essential features of dynamic coupling and a 1:1 resonance. It is relatively simple to construct an experiment, and it is natural for a shallow water fluid configuration. In this paper a model for Cooker's experiment will be studied. 


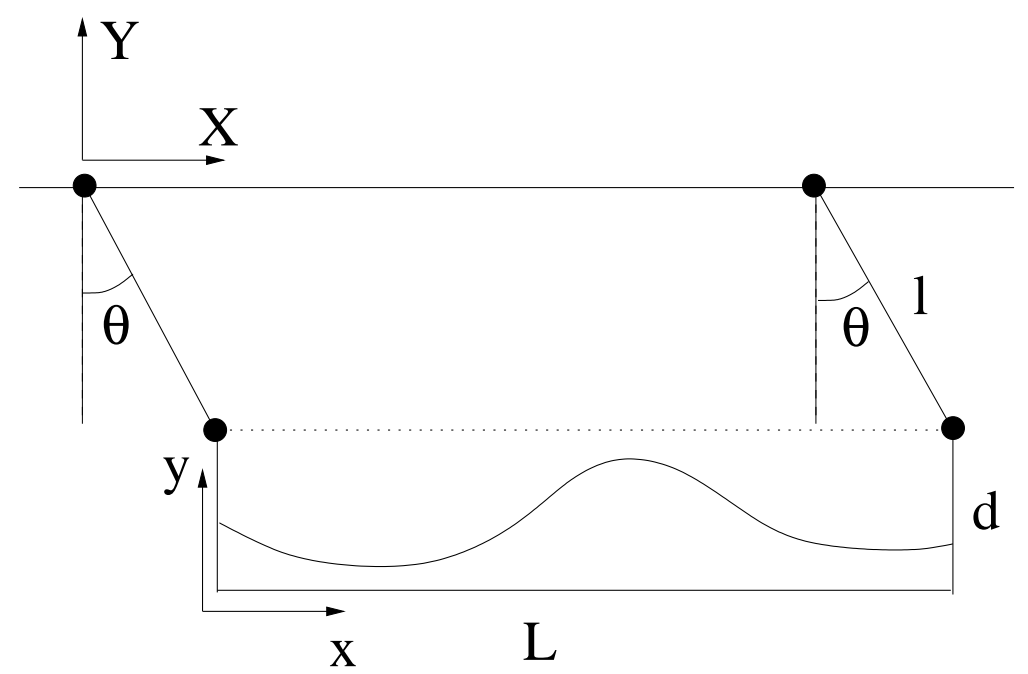

Figure 1. A schematic illustration of Cooker's sloshing experiment.

Cooker (1994) conducted an experiment consisting of a rectangular vessel containing fluid, suspended from a horizontal beam by two cables (or strings). The centre of mass of the system is allowed to rotate in a vertical plane, while the tank bed remains horizontal. A schematic diagram of the experimental apparatus is shown in figure 1 . The vessel has a rectangular cross-section and is partially filled with a fluid of average depth $h_{0}<d$ where $d$ is the vessel height. The suspending strings make an angle $\theta$ with the vertical. The motion of the vessel can be initiated with values of $\theta$ and/or $d \theta / d t$ specified at $t=0$. This configuration is one of the simplest experiments that demonstrates the dynamic coupling between a sloshing fluid and the motion of vessel containing the fluid. The simplicity of the experiment makes it akin to those of Taylor (1974) who would construct simple robust experiments to illuminate and answer some fundamental question in fluid dynamics. A related experiment is that of Herczyński \& Weidman (2012). Using a special low-friction cart, and carefully-controlled initial conditions, they simulated the case where the length of the suspension cables in Cooker's experiment go to infinity. In this case the restoring force vanishes, and so the vessel may drift in addition to exhibiting harmonic motion. They developed a finite depth linear theory for this case and compared their results with experiments, showing excellent agreement for various vessel geometries. However, without the spring force, a resonance between vessel and fluid modes can not arise in this configuration.

Cooker (1994) developed a linear theory for the coupled problem using a shallow water approximation for the fluid, and a linear harmonic equation for the vessel motion. The theoretical results agreed well with Cooker's experimental results which showed that the presence of a sloshing fluid changed the natural frequency of the vessel motion. This theory was extended to include a nonlinear shallow water model by Alemi Ardakani \& Bridges (2010). In his analysis, Cooker (1994) detected a curious resonance where the second mode of the coupled problem appeared to resonate with the natural frequency of the dry vessel. In fact the resonance is a 1:1 internal resonance, where the natural frequency of the vessel, treated as a rigid body, resonates with one of the fluid modes. The precise nature of this internal resonance was identified by Alemi Ardakani et al. $(2012 a, b)$. The key observation is that the characteristic function for the natural frequencies of the coupled problem is the product of two functions

$$
\Delta(\omega)=P(\omega) D(\omega),
$$

where $\omega$ is the frequency of the motion. The precise form of these functions will be presented in $\S 3$. Natural frequencies satisfy $\Delta(\omega)=0$. The roots of $D(\omega)=0$ are the modes which couple the anti-symmetric fluid modes to the vessel motion, while the roots of $P(\omega)=0$ are associated with the symmetric fluid modes (which exert no horizontal force on the vessel). The case when $P(\omega)=D(\omega)=0$ with both $P^{\prime}(\omega)$ and $D^{\prime}(\omega)$ non-zero, produces an internal 1:1 resonance with both types of modes coupled together. The linear aspects of this resonance are studied in detail by Alemi Ardakani et al. $(2012 a, b)$, and it is shown that the 1:1 resonance occurs in the coupled system for a wide range of parameter values.

In the linear problem the 1:1 resonance is benign. There is no instability and it just means that the two modes have the same natural frequency and any linear combination can occur depending on the initial conditions. It is only by adding nonlinearity that dynamic energy transfer can occur. 


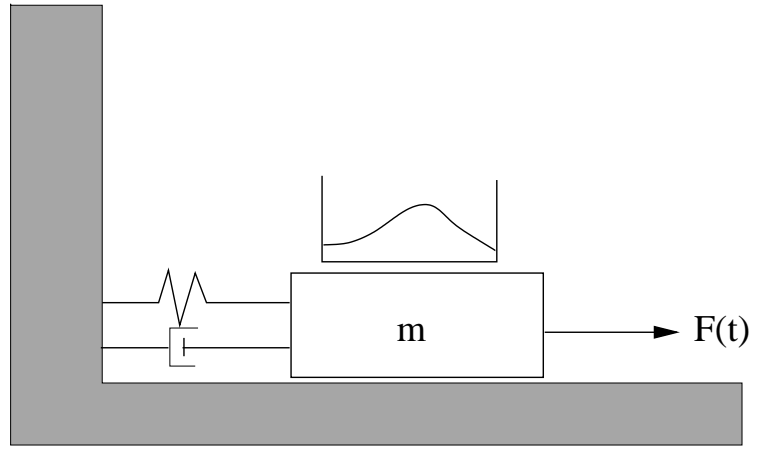

Figure 2. A schematic illustration of a TLD following figure 1 of Frandsen (2005).

Before proceeding to discuss the nonlinear implications of the $1: 1$ resonance, it is worth noting that the problem of tuned liquid dampers (TLDs) (Ikeda \& Nakagawa 1997; Frandsen 2005) has a very similar mathematical formulation. A TLD consists of a vessel partially filled with fluid, but the vessel is constrained to move only in the horizontal direction with a restoring force given by a linear mass-springdamper model. A schematic illustration of this system is given in figure 2. Alemi Ardakani et al. (2012a) showed that the linearized governing equations for the TLD and Cooker's experiment are equivalent, and hence the form of characteristic equation for the linear TLD system, first computed by Frandsen (2005) using an infinite cosine expansion, is equivalent to the characteristic function for the fully-coupled Cooker's experiment, which was first derived by Yu (2010) using a vertical eigenfunction expansion. Although Cooker's experiment and the TLD system are equivalent in the linear framework, their nonlinear characteristics are very different, as the vessel equation described in Alemi Ardakani et al. (2012a) for Cooker's experiment is fully nonlinear and contains vertical as well as horizontal motions. However, the nonlinear analysis presented in this paper could be modified to examine the TLD problem, with the linear spring in Frandsen (2005) replaced by a nonlinear spring, but this extension is not considered here.

Let $A(\tau)$ and $B(\tau)$ be complex modal amplitudes for the two modes in the 1:1 resonance, where $\tau$ is a slow time variable. The nonlinear normal form near the 1:1 resonance is given by

$$
\begin{aligned}
\mathrm{i} a_{0} A_{\tau} & =a_{1} \omega_{n, 2} A+a_{2}|A|^{2} A+a_{3}|B|^{2} A+a_{4} B^{2} A^{*} \\
\mathrm{i} b_{0} B_{\tau} & =b_{1} \omega_{n, 2} B+b_{2}|B|^{2} B+a_{3}|A|^{2} B+a_{4} A^{2} B^{*},
\end{aligned}
$$

where $a_{0}, \ldots, a_{4}, b_{0}, \ldots, b_{2}, \omega_{n, 2}$ are real-valued coefficients where $\omega_{n, 2}$ gives the deviation of the frequency from the sloshing frequency $\omega_{n}$ found via (1.1) and the stars denote the complex conjugate. A derivation of $(1.2)$ is given in $\S 5$.

The nonlinear normal form (1.2) appears in other contexts. The most well-known context is sloshing in three dimensions when the horizontal cross-section of the vessel is square or almost square. This configuration has been studied by Feng \& Sethna (1989); Faltinsen et al. (2003); Ikeda et al. (2012); see also $§ 9.2 .3$ of Faltinsen \& Timokha (2009). There are two significant differences from the analysis here. Firstly, due to the symmetry of the square, the coefficients $a_{2}$ and $b_{2}$ are equal. In that case both the pure modes have the exact same stability properties. Secondly the problem of energy transfer is not considered in the analysis in Feng \& Sethna (1989), Faltinsen et al. (2003) or Ikeda et al. (2012). The analysis of energy transfer is made apparent in the present paper by showing that the orbits of the normal form (1.2) can be projected onto a spheroid in three dimensions.

In order to deduce the physical implications of the 1:1 resonance, it is necessary to compute the coefficients in the normal form (1.2). We compute the coefficients in the normal form for Cooker's experiment as a function of the key dimensionless parameters: depth ratio, cable stiffness ratio, cable length ratio and mass ratio. Although the linear problem has a continuum of periodic solutions (any values of $A$ and $B)$ the nonlinear problem has exactly six: two pure modes $(A=0, B \neq 0$ and $A \neq 0$, $B=0)$ and four mixed modes $(A \neq 0, B \neq 0$, one in phase, three out of phase). Using dynamical systems theory we are able to identify all possible connecting orbits between the periodic solutions. It is these heteroclinic connections that are pathways to energy transfer.

The idea of a linear resonance producing energy transfer in the nonlinear problem has been known in the mechanics literature for over half a century (and perhaps more). A simplified version of Cooker's experiment is to replace the fluid by an elastic beam. This configuration has been studied by Struble 
\& Heinbockel (1963). They identified a $1: 2$ resonance in the linear system, performed a nonlinear analysis and then showed numerically that energy can be exchanged between the two principal modes. The pathway for energy transfer in this case was also a heteroclinic connection. The approach used in this paper, of projecting onto a spheroid, is more general and captures all the potential heteroclinic connections in a given configuration.

It should be noted that energy transfer between modes can also occur without resonance in the nonlinear case, when forcing is included in the problem. One such situation was examined by Feng (1998) who studied the sloshing of one symmetric and one anti-symmetric fluid mode under a time dependent forcing. However, the analysis and the mechanism for the energy transfer were very different to those studied in this paper for the non-forced case.

The current paper is laid out as follows. In $\S 2$ we state the governing nonlinear equations of the system and show that they can be obtained from a Lagrangian functional. Section 3 reviews the linear solution to the problem and highlights the internal 1:1 resonance between the coupled motion of the fluid and the vessel. In $\S 4$ the nonlinear solution to the coupled equations are found by expanding the solution in powers of the eigenmode amplitude and in $\S 5$ the normal form equations are derived. The general stability properties of the normal form equations are formulated in $\S 6$ while energy transfer properties are discussed in $\$ 7$. Section 8 presents numerical results for Cooker's experiment and our concluding remarks are given in $\S 9$. The supplementary material which accompanies this paper presents more details on the perturbation solution procedure and the normal form calculation, as well as a comparison of the pure standing waves (decoupled from the vessel motion) with the classical theory for standing waves of Tadjbakhsh \& Keller (1960).

\section{Governing nonlinear equations}

The governing equations consist of the Euler equations for the fluid, relative to a moving frame, coupled to the rigid-body equations for the vessel motion. The configuration is illustrated in figure 1 . The tank has length $L$ and width $W$, while the fluid has constant density $\rho$ and the suspending string length is $l$. The fixed coordinate system $(X, Y)$ has its origin at the left most fixed point of the suspension string, and the moving coordinates $(x, y)$ are attached to the vessel. These coordinates are related by

$$
X=x+q_{1}, \quad Y=y+q_{2}-d,
$$

where

$$
q_{1}=l \sin \theta(t), \quad q_{2}=-l \cos \theta(t)
$$

which parametrise the constraint $q_{1}^{2}+q_{2}^{2}=l^{2}$. Denote the velocity field relative to the moving frame by $(u(x, y, t), v(x, y, t))$. With the further assumption of irrotational flow the velocity field can be represented in terms of a potential

$$
\phi_{x}=u+\dot{q}_{1} \text { and } \phi_{y}=v+\dot{q}_{2},
$$

where the velocity potential is relative to the absolute frame (cf. equation (2.1) in Alemi Ardakani et al. $(2012 a)$ ). The governing equations for the fluid are then (cf. Alemi Ardakani et al. 2012a,b)

$$
\begin{aligned}
\phi_{x x}+\phi_{y y}=0, & \text { in } 0 \leq x \leq L, 0 \leq y \leq h(x, t), \\
\phi_{t}+\frac{1}{2}\left(\phi_{x}^{2}+\phi_{y}^{2}\right)-\dot{q}_{1} \phi_{x}-\dot{q}_{2} \phi_{y}+g\left(y-h_{0}\right)=B e, & \text { on } y=h(x, t), \\
h_{t}+\left(\phi_{x}-\dot{q}_{1}\right) h_{x}=\phi_{y}-\dot{q}_{2}, & \text { on } y=h(x, t), \\
\phi_{y}=\dot{q}_{2}, & \text { on } y=0, \\
\phi_{x}=\dot{q}_{1}, & \text { on } x=0, L,
\end{aligned}
$$

where $B e$ is a Bernoulli constant. (Note that the Bernoulli constant could be absorbed into $\phi$ as a term proportional to $t$ ). The vessel motion is governed by a nonlinear forced pendulum equation

$$
m_{v} \ddot{\theta}+\frac{g}{l}\left(m_{v}+m_{f}\right) \sin \theta=-\frac{1}{l} \cos \theta \dot{\sigma}_{1}-\frac{1}{l} \sin \theta \dot{\sigma}_{2} .
$$

Here the subscripts $x, y$ and $t$ denote partial derivatives and dots denote full derivatives with respect to $t$. Equations (2.2) and (2.3) are the dynamic and kinematic boundary conditions at the free surface $y=h(x, t),(2.4)$ and $(2.5)$ are the no penetration boundary conditions on the bottom and side walls 
of the vessel and (2.6) is the nonlinear coupling equation linking the motion of the fluid to the vessel motion. The quantities $m_{v}$ and $m_{f}=W \int_{0}^{L} \int_{0}^{h} \rho d y d x$ are the vessel and fluid masses respectively, and

$$
\sigma_{1}(t)=W \int_{0}^{L} \int_{0}^{h} \rho \phi_{x} d y d x, \quad \sigma_{2}(t)=W \int_{0}^{L} \int_{0}^{h} \rho \phi_{y} d y d x .
$$

The key parameters in the problem are

$$
\delta=\frac{h_{0}}{L}, \quad \ell=\frac{L}{l}, \quad R=\frac{m_{v}}{m_{f}}, \quad G=\frac{\nu L^{2}}{4 g h_{0} m_{f}}, \quad \nu=\frac{g}{l}\left(m_{v}+m_{f}\right) .
$$

The parameter $\delta$ is a shallowness parameter, and $G$ is the stiffness parameter, since $\nu$ is an equivalent spring stiffness. The parameters $R$ and $G$ were introduced by Cooker (1994), and the parameters are not all independent since $G$ has the equivalent representation

$$
G=\frac{(1+R)}{4} \frac{\ell}{\delta}
$$

The governing equations are variational, i.e. they can be determined by taking the first variation of a Lagrangian functional. It is useful to use a Lagrangian approach for calculating the normal form. Alemi Ardakani et al. (2012a) defined a reduced Lagrangian for this system in order to derive the coupling equation (2.6), but did not include the constraint terms needed to correctly describe the fluid motion. The complete Lagrangian for this system is

$$
\mathscr{L}=\int_{t_{1}}^{t_{2}} \mathcal{L} d t
$$

where $\left[t_{1}, t_{2}\right]$ is an arbitrary time interval, and

$$
\begin{aligned}
\mathcal{L}=\rho W & \int_{0}^{L} \int_{0}^{h(x, t)}\left(\phi_{t}+\frac{1}{2}\left(\phi_{x}^{2}+\phi_{y}^{2}\right)+g\left(y-h_{0}\right)-B e\right) d y d x \\
& -\frac{1}{2} m_{v} l^{2} \dot{\theta}^{2}-\left(m_{v}+m_{f}\right) g l \cos \theta-l \dot{\theta} \rho W \int_{0}^{L} \int_{0}^{h(x, t)}\left(\phi_{x} \cos \theta+\phi_{y} \sin \theta\right) d y d x,
\end{aligned}
$$

where $h_{0}$ is the mean fluid height. The precise form of this Lagrangian is new, but the idea of using a Lagrangian to generate the fluid equations for sloshing has a long history. See $§ 2.5 .3$ of Faltinsen \& Timokha (2009) for a history of the use of the Lagrangian formalism for sloshing.

The Lagrangian functional (2.10) has three parts. The first part is Luke's Lagrangian for the fluid motion only (Luke 1967), the second part is the Lagrangian for the vessel and the third term is the coupling term. By taking variations of (2.9)-(2.10) with respect to $\phi, h$ and $\theta$ the governing equations (2.1)-(2.6) are recovered.

\section{Solution of the linear coupled problem}

The solution to the linear coupled fluid-vessel system has been studied in detail in both the shallow water limit and in finite depth by Alemi Ardakani et al. (2012b). Here we will just record the details necessary for the nonlinear analysis to follow.

The governing equations for the linear motion are found by linearizing (2.1)-(2.6) about a quiescent fluid level $h_{0}, \theta=0$ and $\phi=$ constant. The linear equations are

$$
\begin{aligned}
\phi_{x x}+\phi_{y y}=0, & \text { in } 0 \leq x \leq L, 0 \leq y \leq h_{0}, \\
\phi_{t t}+g \phi_{y}=0 & \text { on } y=h_{0} \\
\phi_{y}=0, & \text { on } y=0 \\
\phi_{x}=l \dot{\theta}, & \text { on } x=0, L
\end{aligned}
$$

for the fluid motion, where the second equation is obtained by combining the linear kinematic and dynamic free surface conditions

$$
\phi_{t}+g h=0 \text { and } h_{t}=\phi_{y} \Longrightarrow \phi_{t t}+g \phi_{y}=0 .
$$


The linear vessel motion is governed by

$$
m_{v} \ddot{\theta}+\frac{g}{l}\left(m_{v}+m_{f}\right) \theta=-\frac{W}{l} \int_{0}^{L} \int_{0}^{h_{0}} \rho \phi_{x t} d y d x .
$$

Time-periodic solutions of these equations can be found by expressing $\phi, h$ and $\theta$ as the harmonic functions

$$
\phi(x, y, t)=\hat{\phi}(x, y) \cos \omega t, \quad h(x, t)=\hat{h}(x) \sin \omega t, \quad \theta(t)=\hat{\theta} \sin \omega t .
$$

The above system of equations are solved using either of the following two approaches. The first approach is to expand $\hat{\phi}$ as an infinite cosine expansion (Graham \& Rodriguez 1952; Frandsen 2005; Herczyński \& Weidman 2012), while the second approach is to expand $\hat{\phi}$ as an infinite sum of vertical eigenfunctions (Linton \& McIver 2001; Yu 2010; Alemi Ardakani et al. 2012a). Alemi Ardakani et al. $(2012 a, b)$ show that both expansions are equivalent, so in theory either approach can be used. However, in practice the cosine expansion is more accurate when performing the numerical calculations required in this paper. Thus this method is used in the results reported herein, although it should be noted that the sums converge slower than the sums in the vertical eigenfunction expansion.

The characteristic function for the linear problem is given by the product (1.1) with

$$
\begin{aligned}
& P(\omega)=\prod_{n=1}^{\infty}\left(\omega^{2}-g \beta_{n} \tanh \left(\beta_{n} h_{0}\right)\right) \\
& D(\omega)=\left(m_{v}+m_{f}\right)\left(\frac{g}{\omega}-l \omega\right)-\frac{8 l m_{f} \omega^{3}}{L^{2} h_{0}} \sum_{m=0}^{\infty} \frac{\tanh \left(\alpha_{m} h_{0}\right)}{\alpha_{m}^{3}\left(g \alpha_{m} \tanh \left(\alpha_{m} h_{0}\right)-\omega^{2}\right)},
\end{aligned}
$$

where

$$
\beta_{n}=\frac{2 n \pi}{L}, \quad \alpha_{m}=\frac{(2 m+1) \pi}{L} .
$$

The solutions of $P(\omega)=0$ correspond to contributions from symmetric sloshing modes with an $x$-dependence of the form $\cos \beta_{n} x$, while $D(\omega)=0$ contains contributions from the anti-symmetric sloshing modes with an $x$-dependence of the form $\cos \alpha_{m} x$.

In this work we are interested in the nonlinear solution for the system near the 1:1 resonance, which occurs at a double zero of the dispersion relation (1.1). If we assume that this resonance occurs with a general symmetric mode with wavenumber $\beta_{n}$, then the frequency of the coupled solution is given by

$$
\omega_{n}^{2}=g \beta_{n} \tanh \left(\beta_{n} h_{0}\right)
$$

The results will be presented with $n$ an arbitrary natural number, but $n=1$ is the case most likely to be observed in experiments. As the 1:1 resonance occurs at a double zero of (1.1) this also requires that $D\left(\omega_{n}\right)=0$. This allows for the calculation of the string length $l$, say, needed for resonance for the given parameters $m_{v}, m_{f}, h_{0}$ and $L$. Substituting $(3.8)$ into $D(\omega)=0$ gives the condition on the parameters for $1: 1$ resonance

$$
G=s_{n}^{2} R+s_{n}^{2}+\frac{8}{\delta} s_{n}^{4} \sum_{m=0}^{\infty} \frac{\tanh ((2 m+1) \pi \delta)}{(2 m+1)^{3} \pi^{3} \sigma_{m, n}}
$$

where

$$
s_{n}^{2}=\frac{\omega_{n}^{2} L^{2}}{4 g h_{0}} \quad \text { and } \quad \sigma_{m, n}=g \alpha_{m} \tanh \left(\alpha_{m} h_{0}\right)-\omega_{n}^{2} .
$$

For each fixed $n$ and $\delta$, the condition (3.9) is a line in the $(R, G)$-plane.

At the 1:1 resonance the solution for $\hat{\phi}$ is made up of two linearly independent eigenfunctions in the form

$$
\hat{\phi}=\widetilde{A} S_{0}(x, y)+\widetilde{B} S_{1}(x, y),
$$

(cf. $§ 4$ of Alemi Ardakani et al. 2012a) where

$$
\begin{aligned}
S_{0}(x, y) & =\frac{\cosh \left(\beta_{n} y\right)}{\cosh \left(\beta_{n} h_{0}\right)} \cos \left(\beta_{n} x\right) \\
S_{1}(x, y) & =l \omega_{n}\left(x-\frac{1}{2} L-\frac{4 \omega_{n}^{2}}{L} \sum_{m=0}^{\infty} \frac{1}{\alpha_{m}^{2} \sigma_{m, n}} \frac{\cosh \left(\alpha_{m} y\right)}{\cosh \left(\alpha_{m} h_{0}\right)} \cos \left(\alpha_{m} x\right)\right)
\end{aligned}
$$


and $\hat{\theta}$ has now been replaced by $\widetilde{B}$. The solution (3.11) consists of a linear combination of symmetric sloshing modes, $S_{0}(x, y)$, with amplitude $\widetilde{A}$ and anti-symmetric sloshing modes, $S_{1}(x, y)$, with amplitude $\widetilde{B}$. The symmetric sloshing modes are observed when sloshing occurs in a stationary vessel, while the anti-symmetric modes are observed when sloshing occurs with intrinsic coupling to the moving vessel.

\section{Nonlinear analysis}

In this section we perform a perturbation analysis of the nonlinear equations of motion (2.1)-(2.6) close to the resonance frequency $\omega_{n}$. This solution will then be used in (2.9) to give the form of the Lagrangian, from which we can generate the nonlinear normal form of the sloshing problem at an internal 1:1 resonance.

In order to generate the nonlinear correction terms to the linear eigenfunction, we assume that the amplitude of the linear solution is small and expand the solution in terms of this small parameter. Thus we assume that $\widetilde{A}=\epsilon A$ and $\widetilde{B}=\epsilon B$ where $\epsilon \ll 1$, and we also introduce a scaled time $\widetilde{t}$ and a slow time scale $\tau$ defined by

$$
\widetilde{t}=\omega t \text { and } \tau=\epsilon^{2} t .
$$

The solutions are sought which are $2 \pi$-periodic in $\widetilde{t}$. Since it will be clear from the context, the ${ }^{\sim}$ is dropped from the time and it is understood that solutions are now $2 \pi$-periodic in $t$. Substituting

$$
\begin{aligned}
\phi & =\Phi_{0}+\epsilon \phi_{1}(x, y, t, \tau)+\epsilon^{2} \phi_{2}(x, y, t, \tau)+O\left(\epsilon^{3}\right), \\
h & =h_{0}+\epsilon h_{1}(x, t, \tau)+\epsilon^{2} h_{2}(x, t, \tau)+O\left(\epsilon^{3}\right), \\
\theta & =\epsilon \theta_{1}(t, \tau)+\epsilon^{2} \theta_{2}(t, \tau)+O\left(\epsilon^{3}\right), \\
\omega & =\omega_{n}+\epsilon \omega_{n, 1}+\epsilon^{2} \omega_{n, 2}+O\left(\epsilon^{3}\right), \\
B e & =\epsilon^{2} B e_{2},
\end{aligned}
$$

into (2.1)-(2.6), where $\Phi_{0}$ is a constant, leads to the linearised form of the equations (3.1)-(3.5) at $O(\epsilon)$ and at $O\left(\epsilon^{2}\right)$ gives the following system of equations for $\phi_{2}, h_{2}$ and $\theta_{2}$ :

$$
\begin{aligned}
\phi_{2 x x}+\phi_{2 y y} & =0 \text { in } 0<y<h_{0}, 0<x<L, \\
\omega_{n} \phi_{2 t}+g h_{2} & =B e_{2}-\omega_{n} h_{1} \phi_{1 y t}-\frac{1}{2}\left(\phi_{1 x}^{2}+\phi_{1 y}^{2}\right)+l \omega_{n} \theta_{1 t} \phi_{1 x}-\frac{1}{2} l^{2} \omega_{n}^{2} \theta_{1 t}^{2}-\omega_{n, 1} \phi_{1 t} \text { on } y=h_{0}, \\
\omega_{n} h_{2 t} & =\phi_{2 y}-\left(\phi_{1 x}-l \omega_{n} \theta_{1 t}\right) h_{1 x}+h_{1} \phi_{1 y y}-l \omega_{n} \theta_{1} \theta_{1 t}-\omega_{n, 1} h_{1 t} \text { on } y=h_{0}, \\
\phi_{2 y} & =l \omega_{n} \theta_{1} \theta_{1 t} \text { on } y=0, \\
\phi_{2 x} & =l \omega_{n} \theta_{2 t}+l \omega_{n, 1} \theta_{1 t} \text { on } x=0, L,
\end{aligned}
$$

for the fluid motion and

$$
\begin{aligned}
m_{v} \omega_{n}^{2} \theta_{2 t t}+\frac{g}{l}\left(m_{v}+m_{f}\right) \theta_{2}=- & 2 m_{v} \omega_{n} \omega_{n, 1} \theta_{1 t t}-\frac{\omega_{n} W}{l} \int_{0}^{L} \int_{0}^{h_{0}} \rho \phi_{2 x t} d y d x-\frac{\omega_{n} \theta_{1} W}{l} \int_{0}^{L} \int_{0}^{h_{0}} \rho \phi_{1 y t} d y d x \\
& -\frac{\omega_{n} W}{l} \int_{0}^{L} \rho h_{1 t} \phi_{1 x}\left(x, y=h_{0}, t\right) d x-\frac{\omega_{n} W}{l} \int_{0}^{L} \rho h_{1} \phi_{1 x t}\left(x, y=h_{0}, t\right) d x \\
& -\frac{\omega_{n, 1} W}{l} \int_{0}^{L} \int_{0}^{h_{0}} \rho \phi_{1 x t} d y d x
\end{aligned}
$$

for the vessel. The dynamic and kinematic free-surface conditions (4.3) and (4.4) can be combined together to give the single free-surface condition

$$
\begin{aligned}
\omega_{n}^{2} \phi_{2 t t}+g \phi_{2 y}=\quad & 2 \omega_{n} \phi_{1 x} \phi_{1 t x}+\omega_{n} \phi_{1 t} \phi_{1 y y}-2 \omega_{n} \phi_{1 y} \phi_{1 t y}+\omega_{n}^{3} g^{-1} \phi_{1 t} \phi_{1 y t t}+2 l \omega_{n}^{2} \theta_{1 t} \phi_{1 x t} \\
& +l \omega_{n}^{2} \theta_{1 t t} \phi_{1 x}+g l \omega_{n} \theta_{1} \theta_{1 t}-l^{2} \omega_{n}^{3} \theta_{1 t} \theta_{1 t t}-2 \omega_{n} \omega_{n, 1} \phi_{1 t t} .
\end{aligned}
$$

By applying a solvability condition to the above system of equations we find that $\omega_{n, 1}=0$ (see appendix A of the supplementary material for detailed calculation). With $\omega_{n, 1}=0$ it is clear that a solution for $\phi_{2}$ and $\theta_{2}$ should be sought in the form

$$
\phi_{2}(x, y, t)=\hat{\phi}_{2}(x, y) \sin (2 t), \quad \theta_{2}(t)=\hat{\theta}_{2} \cos (2 t) .
$$

The details of the solution procedure for $\hat{\phi}_{2}$ and $\hat{\theta}_{2}$ are given in appendix $\mathrm{A}$ and the final solutions take 


\begin{tabular}{ll}
\hline Parameter & Value \\
\hline$m_{v}$ & $0.552 \mathrm{~kg}$ \\
$W$ & $0.13 \mathrm{~m}$ \\
$L$ & $0.525 \mathrm{~m}$ \\
$\rho$ & $1000 \mathrm{kgm}^{-3}$ (water)
\end{tabular}

TABLE 1. Values of the parameters used in this paper, based on the experiments in Cooker (1994).

the form

$$
\begin{aligned}
& \hat{\phi}_{2}=A^{2}\left(\zeta_{0}(x, y)+Z_{3}\right)+A B \zeta_{1}(x, y)+B^{2}\left(\zeta_{2}(x, y)+Z_{1} y+Z_{2}\right), \\
& \hat{\theta}_{2}=A B \Theta_{1},
\end{aligned}
$$

where the $\zeta_{i}$ 's, $Z_{i}$ 's and $\Theta_{1}$ are given by (A 8)-(A 14). In order to find the form of $h_{2}(x, t)$, the complex exponential form of the solution to $(2.1)$ to $(2.6)$ is used so that phase effects are included. This is discussed in the next section, where we derive the normal form of the nonlinear perturbation about the 1:1 internal resonance. The asymptotic expansions used in the above analysis are valid, assuming that no other resonances occur in the problem.

Although we are interested in the nonlinear result close to the internal resonance in this paper, the solution (4.10) also gives the weakly nonlinear form of the solution away from resonance. At resonance, the two constants $A$ and $B$ are independent and typically (for general initial conditions) both are nonzero, while away from resonance only the pure modes can exist as solutions with either $A=0$ or $B=0$. For the case when $A=0$ with $B \neq 0$ the value of $\hat{\omega}$ is found by solving $D(\hat{\omega})=0$ from (3.7). Here we write $\hat{\omega}$ rather than $\omega_{n}$ because we are away from resonance, so the frequency of the solution is now not determined by (3.8), but by the solution of $D(\hat{\omega})=0$. The nonlinear form of $h(x, t)$ (from $(5.2)$ ) for the lowest frequency sloshing mode not at resonance $(\widetilde{A}=\epsilon A=0, \widetilde{B}=\epsilon B=0.1)$ is shown in figure 3 and the profile of the lowest frequency sloshing mode at resonance $\left(\widetilde{A}=0.015 \mathrm{~m}^{2} \mathrm{~s}^{-1}\right.$ and $\left.\widetilde{B}=0.01\right)$ is shown in figure 4. Note that the numerical results presented in this paper are solved with the parameter values given in table 1, which correspond to the series of experiments of Cooker (1994).

The effect of the nonlinear terms in figures 3 and 4 is to add higher order harmonics to the free surface height. This can be seen in figure $3(\mathrm{c})$ around $x=0.45 \mathrm{~m}$ where the free surface has a region where it decreases before rising up to the wall. The excitation of higher harmonics can be more clearly seen in figure $4(\mathrm{c})$ at $x=0.3 \mathrm{~m}$ where the nonlinear free surface has two local minima compared to the dashed line of the linear result which has a single minimum value close to this point.

The nonlinear results away from the internal 1:1 resonance presented here are new, but it is the nonlinear results close to the 1:1 resonance which are of most interest, along with the normal form found using this solution which is calculated in the next section. The nonlinear normal form near this resonance will enable us to determine parameter regimes where energy transfer between the pure modes is possible.

Before deriving the normal form in the next section, we note that the solution (4.10) when restricted to a pure fluid mode recovers the perturbation solution calculated by Tadjbakhsh \& Keller (1960). They consider the nonlinear expansion about the first symmetric sloshing mode only $(B=0$ with $n=1)$. The details of this comparison with classical standing waves is in appendix B of the supplementary material.

\section{Normal form equations}

In order to include phase effects, $A$ and $B$ are now taken to be complex. The strategy for computing the coefficients of the normal form (1.2) is to substitute the expansions for $\phi, h$ and $\theta$ up to second order in $\epsilon$ into the Lagrangian (2.9), and then take variations with respect to $A^{*}$ and $B^{*}$ where the $*$ here 

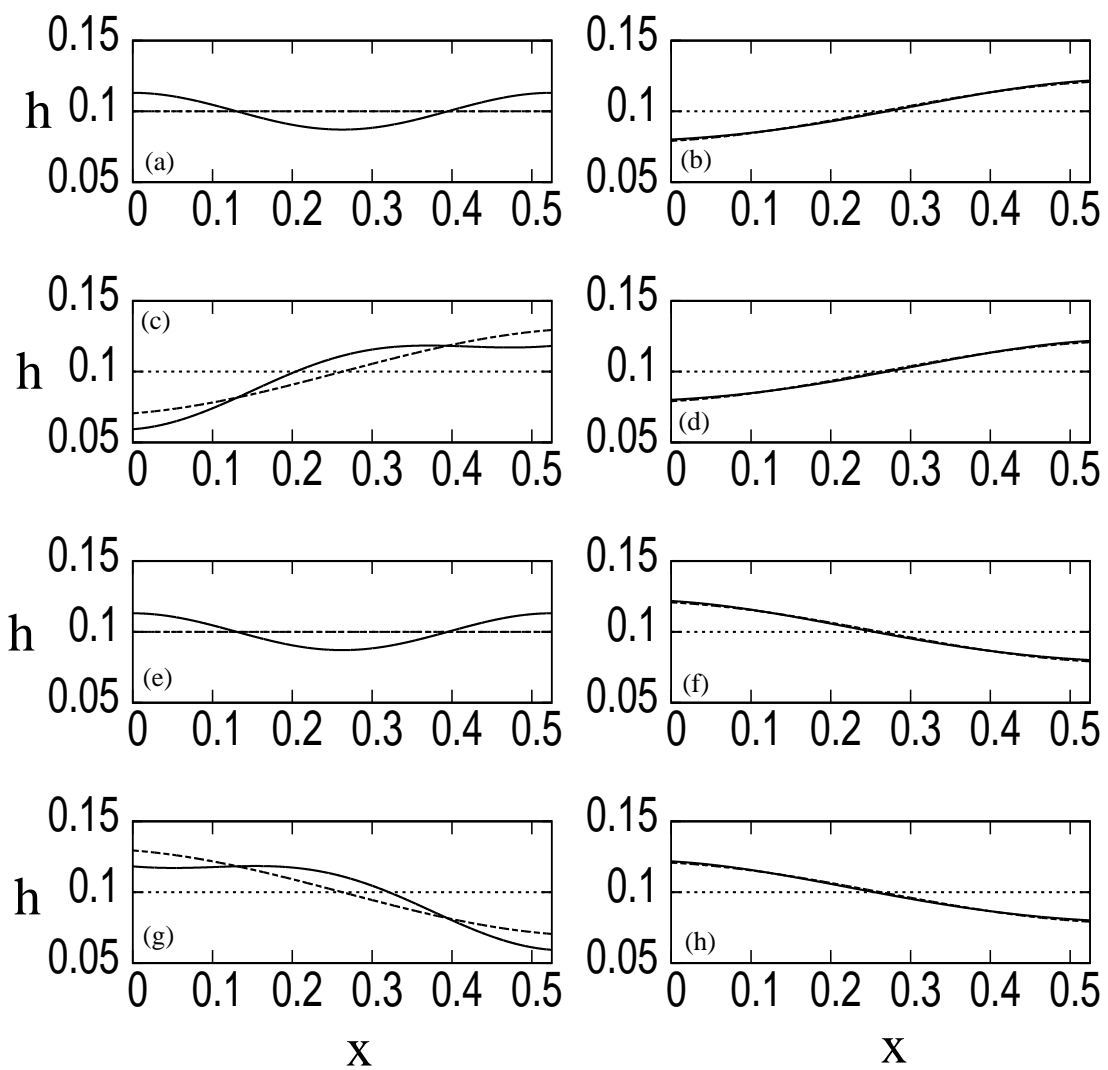

FiguRE 3. Plot of $h(x, t)$ for the lowest frequency sloshing mode not at resonance over one period of the motion, when $h_{0}=0.1 \mathrm{~m}, l=0.2 \mathrm{~m}$ and $\hat{\omega}=4.575 \mathrm{~s}^{-1}$. Here $\widetilde{A}=0$ and $\widetilde{B}=0.1$. In each panel the solid line gives the nonlinear result up to, and including, second order terms, the dashed line gives the linear result and the dotted line gives the value of $h_{0}$.

denotes the complex conjugate. The forms of $\phi, h$ and $\theta$ to second order are

$$
\begin{aligned}
\phi(x, y, t, \tau)= & \Phi_{0}+\frac{1}{2} \epsilon\left[\left(A S_{0}(x, y)+B S_{1}(x, y)\right) e^{\mathrm{i} t}+\left(A^{*} S_{0}(x, y)+B^{*} S_{1}(x, y)\right) e^{-\mathrm{i} t}\right] \\
& -\frac{1}{2} \epsilon^{2} \mathrm{i}\left[\left(A^{2} \zeta_{0}(x, y)+A B \zeta_{1}(x, y)+B^{2} \zeta_{2}(x, y)+B^{2} Z_{1} y+B^{2} Z_{2}+A^{2} Z_{3}\right) e^{2 \mathrm{i} t}\right. \\
& \left.-\left(A^{* 2} \zeta_{0}(x, y)+A^{*} B^{*} \zeta_{1}(x, y)+B^{* 2} \zeta_{2}(x, y)+B^{* 2} Z_{1} y+B^{* 2} Z_{2}+A^{* 2} Z_{3}\right) e^{-2 \mathrm{i} t}\right] \\
& +O\left(\epsilon^{3}\right), \\
h(x, t, \tau)= & h_{0}-\frac{1}{2} \epsilon \mathrm{i}\left[\left(A H_{0}(x)+B H_{1}(x)\right) e^{\mathrm{i} t}-\left(A^{*} H_{0}(x)+B^{*} H_{1}(x)\right) e^{-\mathrm{i} t}\right] \\
& +\frac{1}{2} \epsilon^{2}\left[\left(A^{2} \lambda_{0}(x)+A B \lambda_{1}(x)+B^{2} \lambda_{2}(x)\right) e^{2 \mathrm{i} t}+\left(A^{* 2} \lambda_{0}(x)+A^{*} B^{*} \lambda_{1}(x)+B^{* 2} \lambda_{2}(x)\right) e^{-2 \mathrm{i} t}\right. \\
& \left.+\left(\Lambda_{0}(x)-C_{0}\right)|A|^{2}+\left(\Lambda_{1}(x)-C_{1}\right) A B^{*}+\left(\Lambda_{2}(x)-C_{2}\right) A^{*} B+\left(\Lambda_{3}(x)-C_{3}\right)|B|^{2}\right] \\
& +O\left(\epsilon^{3}\right), \\
\theta(t, \tau)= & -\frac{1}{2} \epsilon \mathrm{i}\left[B e^{\mathrm{i} t}-B^{*} e^{-\mathrm{i} t]}+\frac{1}{2} \epsilon^{2}\left[A B \Theta_{1} e^{2 \mathrm{i} t}+A^{*} B^{*} \Theta_{1} e^{-2 \mathrm{i} t}\right]+O\left(\epsilon^{3}\right)\right. \\
B e= & -\frac{1}{2} \epsilon^{2} g\left(C_{0}|A|^{2}+C_{1} A B^{*}+C_{2} A^{*} B+C_{3}|B|^{2}\right) \\
&
\end{aligned}
$$

where $S_{0}$ and $S_{1}$ are given in (3.11), the $\zeta_{i}$ 's, $Z_{i}$ 's and $\Theta_{1}$ are given in Appendix A and the constants $C_{0}$ to $C_{3}$ are determined by

$$
C_{i}=\frac{1}{L} \int_{0}^{L} \Lambda_{i}(x) d x
$$



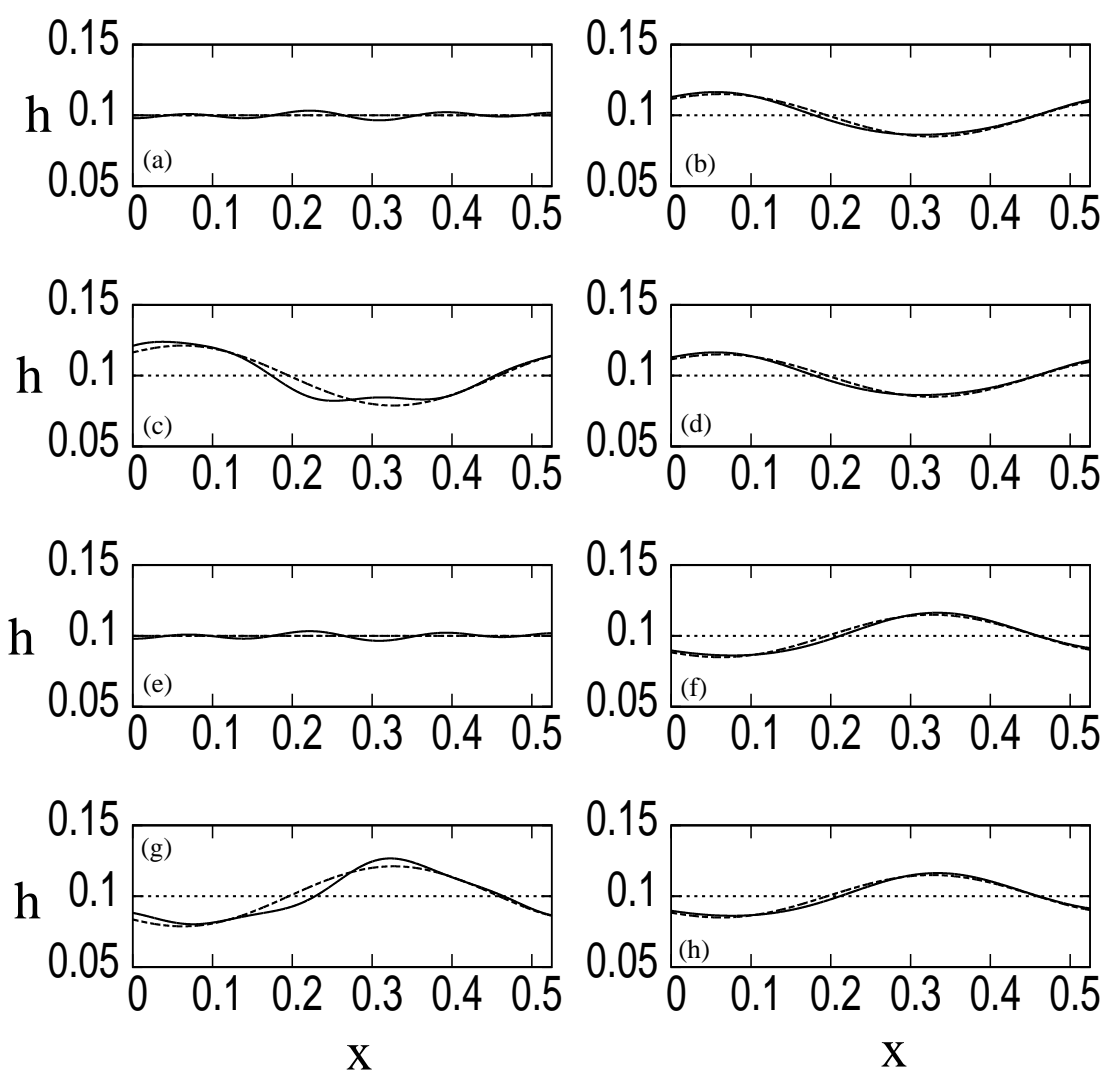

Figure 4. Plot of $h(x, t)$ for the lowest frequency sloshing mode at resonance over one period of the motion, when $h_{0}=0.1 \mathrm{~m}, l=1.270 \mathrm{~m}$ and $\omega_{1}=9.341 \mathrm{~s}^{-1}$. Here $\widetilde{A}=0.015 \mathrm{~m}^{2} \mathrm{~s}^{-1}$ and $\widetilde{B}=0.01$. In each panel the solid line gives the nonlinear result up to, and including, second order terms, the dashed line gives the linear result and the dotted line gives the value of $h_{0}$.

The expressions $H_{0}(x), H_{1}(x)$ along with the $\lambda_{i}$ 's and $\Lambda_{i}$ 's are to be determined. The definitions of $C_{i}$ 's constrains the mean height of the fluid to remain at $y=h_{0}$. Not incorporating these constants into the expression for $\phi(x, y, t, \tau)$ would cause the mean height of the fluid to change, which in turn would modify the value of $m_{f}$. Thus for consistency these constants are moved into the expansion for $\phi(x, y, t, \tau)$.

The amplitudes $\widetilde{A}=\epsilon A$ and $\widetilde{B}=\epsilon B$ are now functions of the slow time variable $\tau=\epsilon^{2} t$. The functions $H_{0}, H_{1}$, the $\lambda_{i}$ 's and the $\Lambda_{i}$ 's can be found by either substituting the above expressions into (3.2) and (4.3) and equating the coefficients of $A, B, A^{2}, B^{2}$ and $A B$ or by substituting directly into (2.2). Both 
approaches lead to the functions

$$
\begin{aligned}
H_{0}(x) & =\frac{\omega_{n}}{g} S_{0}, \\
H_{1}(x) & =\frac{\omega_{n}}{g} S_{1} \\
\lambda_{0}(x) & =-\frac{1}{4 g}\left(S_{0 x}^{2}+S_{0 y}^{2}+2 \omega_{n} H_{0} S_{0 y}+8 \omega_{n} \zeta_{0}+8 \omega_{n} Z_{3}\right), \\
\lambda_{1}(x) & =-\frac{1}{2 g}\left(S_{0 x} S_{1 x}+S_{0 y} S_{1 y}+\omega_{n} H_{0} S_{1 y}+\omega_{n} H_{1} S_{0 y}-l \omega_{n} S_{0 x}+4 \omega_{n} \zeta_{1}\right), \\
\lambda_{2}(x) & =-\frac{1}{4 g}\left(S_{1 x}^{2}+S_{1 y}^{2}+2 \omega_{n} H_{1} S_{1 y}-2 l \omega_{n} S_{1 x}+8 \omega_{n} \zeta_{2}+8 \omega_{n} Z_{1} h_{0}+8 \omega_{n} Z_{2}\right), \\
\Lambda_{0}(x) & =-\frac{1}{2 g}\left(S_{0 x}^{2}+S_{0 y}^{2}-2 \omega_{n} H_{0} S_{0 y}\right), \\
\Lambda_{1}(x)= & =-\frac{1}{2 g}\left(S_{0 x} S_{1 x}+S_{0 y} S_{1 y}-\omega_{n} H_{0} S_{1 y}-\omega_{n} H_{1} S_{0 y}-l \omega_{n} S_{0 x}\right), \\
\Lambda_{3}(x) & =-\frac{1}{2 g}\left(S_{1 x}^{2}+S_{1 y}^{2}-2 \omega_{n} H_{1} S_{1 y}-2 l \omega_{n} S_{1 x}\right),
\end{aligned}
$$

where $S_{0}, S_{1}, \zeta_{0}, \zeta_{1}$ and $\zeta_{2}$ are all evaluated at $y=h_{0}$. From these expressions it is immediately clear that $C_{1}=C_{2}=0$, and in fact

$$
\begin{aligned}
& C_{0}=-\frac{\beta_{n}^{2}}{4 g}\left(1-T_{n}^{2}\right), \\
& C_{3}=\frac{l^{2} \omega_{n}^{2}}{2 g}\left(1-\frac{8 \omega_{n}^{4}}{L^{2}} \sum_{m=0}^{\infty} \frac{1-\bar{T}_{m}^{2}}{\alpha_{m}^{2} \sigma_{m, n}^{2}}\right),
\end{aligned}
$$

where $T_{n}=\tanh \left(\beta_{n} h_{0}\right)$ and $\bar{T}_{m}=\tanh \left(\alpha_{m} h_{0}\right)$.

Substituting these expressions into (2.9), setting $t_{1}=0, t_{2}=2 \pi$ and noting that we require a further integral in the Lagrangian as we have introduced the slow timescale $\tau$, we find that

$$
\begin{aligned}
\mathscr{L}=\epsilon^{3} & \int_{\tau_{1}}^{\tau_{2}}\left(\frac{1}{2} \mathrm{i} a_{0}\left(A^{*} A_{\tau}-A A_{\tau}^{*}\right)+\frac{1}{2} \mathrm{i} b_{0}\left(B^{*} B_{\tau}-B B_{\tau}^{*}\right)-a_{1} \omega_{n, 2}|A|^{2}-b_{1} \omega_{n, 2}|B|^{2}\right. \\
& \left.-\frac{1}{2} a_{2}|A|^{4}-\frac{1}{2} b_{2}|B|^{4}-a_{3}|A|^{2}|B|^{2}-\frac{1}{2} a_{4}\left(B^{2} A^{* 2}+A^{2} B^{* 2}\right)\right) d \tau+o\left(\epsilon^{3}\right),
\end{aligned}
$$

where the coefficients $a_{i}$ and $b_{i}$ are given in appendix B.

By taking variations of $\mathscr{L}$ with respect to $A^{*}$ and $B^{*}$ respectively gives the normal form equations to leading order in (1.2). The normal form (1.2) is a Hamiltonian system,

$$
\mathrm{i} a_{0} A_{\tau}=\frac{\delta H}{\delta A^{*}} \quad \text { and } \quad \mathrm{i} b_{0} B_{\tau}=\frac{\delta H}{\delta B^{*}},
$$

where

$$
H=\omega_{n, 2} a_{1}|A|^{2}+\omega_{n, 2} b_{1}|B|^{2}+\frac{1}{2} a_{2}|A|^{4}+\frac{1}{2} b_{2}|B|^{4}+a_{3}|A|^{2}|B|^{2}+\frac{1}{2} a_{4}\left(B^{2} A^{* 2}+A^{2} B^{* 2}\right) .
$$

The Hamiltonian formulation will be useful for identifying heteroclinic connections between periodic solutions. A necessary condition for two periodic solutions to be connected by a heteroclinic orbit is that they have the same energy value (or the same $H$ value). Hence, the energy values for each solution will be recorded.

\section{Solutions of the normal form equations}

Periodic solutions of the original problem are represented by equilibrium solutions of the normal form (1.2). Let

$$
A=r_{A} \mathrm{e}^{\mathrm{i} \mu_{A}} \quad \text { and } \quad B=r_{B} \mathrm{e}^{\mathrm{i} \mu_{B}},
$$


with $r_{A}>0$ and $r_{B}>0$. Then equilibrium solutions satisfy

$$
\begin{aligned}
& 0=\left(a_{1} \omega_{n, 2}+a_{2} r_{A}^{2}+\left(a_{3}+a_{4} \cos \chi\right) r_{B}^{2}\right) r_{A}, \\
& 0=\left(b_{1} \omega_{n, 2}+b_{2} r_{B}^{2}+\left(a_{3}+a_{4} \cos \chi\right) r_{A}^{2}\right) r_{B},
\end{aligned}
$$

with

$$
\sin \chi=0 \quad \text { where } \quad \chi=2\left(\mu_{B}-\mu_{A}\right) .
$$

For non-degenerate values of the coefficients, there are six classes of solutions of these equations.

$$
\text { 6.1. } A \neq 0, B=0 \text {; symmetric free sloshing mode }
$$

When $B=0, A \neq 0$ and $a_{2} \neq 0$, the equations (6.2) reduce to

$$
r_{A}^{2}=-\frac{a_{1}}{a_{2}} \omega_{n, 2}
$$

with stability exponent

$$
\lambda^{2}=\frac{\omega_{n, 2}^{2}}{b_{0}^{2} a_{2}^{2}}\left[a_{1}^{2} a_{4}^{2}-\left(b_{1} a_{2}-a_{1} a_{3}\right)^{2}\right] .
$$

The pure mode (6.4) is stable (unstable) if $\lambda^{2}$ in (6.5) is negative (positive). A derivation of the stability properties is given in Appendix C. The energy value for this class is

$$
H_{A}=-\frac{\omega_{n, 2}^{2} a_{1}^{2}}{2 a_{2}} .
$$

This solution is a pure sloshing mode which is symmetric about the centerline of the vessel, and the vessel is stationary. The eigenfunction to leading order is given by (5.1)-(5.3) with $B=0$. In this case the sloshing modes exert no resultant force on the walls of the vessel and so the vessel remains at rest.

\section{2. $A=0, B \neq 0$; anti-symmetric fluid mode coupled to vessel motion}

When $A=0, B \neq 0$ and $b_{2} \neq 0$, the equations (6.2) reduce to

$$
r_{B}^{2}=-\frac{b_{1}}{b_{2}} \omega_{n, 2}
$$

with stability exponent

$$
\lambda^{2}=\frac{\omega_{n, 2}^{2}}{a_{0}^{2} b_{2}^{2}}\left[b_{1}^{2} a_{4}^{2}-\left(a_{1} b_{2}-b_{1} a_{3}\right)^{2}\right] .
$$

The energy value for this class is

$$
H_{B}=-\frac{\omega_{n, 2}^{2} b_{1}^{2}}{2 b_{2}} .
$$

This solution is a coupled mode, consisting of a fluid sloshing mode which is anti-symmetric about the centerline of the vessel, and the vessel motion is periodic. The eigenfunction to leading order is given by (5.1)-(5.3) with $A=0$. In this case the sloshing modes exert a non-zero resultant force on the vessel walls and so the vessel oscillates.

$$
\text { 6.3. } A \neq 0, B \neq 0 \text {; fully coupled with } \mu_{B}=\mu_{A} \text { or } \mu_{B}=\mu_{A}+\pi
$$

When $A \neq 0$ and $B \neq 0$ the equations (6.2) reduce to

$$
\left[\begin{array}{cc}
a_{2} & a_{3}+a_{4} \\
a_{3}+a_{4} & b_{2}
\end{array}\right]\left(\begin{array}{c}
r_{A}^{2} \\
r_{B}^{2}
\end{array}\right)=-\omega_{n, 2}\left(\begin{array}{l}
a_{1} \\
b_{1}
\end{array}\right) .
$$

This equation is uniquely solvable for $r_{A}^{2}$ and $r_{B}^{2}$ if

$$
a_{2} b_{2}-\left(a_{3}+a_{4}\right)^{2} \neq 0 .
$$

However, both $r_{A}^{2}$ and $r_{B}^{2}$ must be positive so the fixed point to exist. The stability exponent in this case is

$$
\lambda^{2}=4 a_{4} r_{A}^{2} r_{B}^{2}\left(\frac{a_{2}}{a_{0}^{2}}+\frac{b_{2}}{b_{0}^{2}}-2 \frac{a_{3}+a_{4}}{a_{0} b_{0}}\right)
$$


and the energy value for this class is

$$
H_{A B}=-\frac{1}{2} \omega_{n, 2}^{2} \frac{b_{2} a_{1}^{2}+a_{2} b_{1}^{2}-2 a_{1} b_{1}\left(a_{3}+a_{4}\right)}{a_{2} b_{2}-\left(a_{3}+a_{4}\right)^{2}} .
$$

This solution is a fully coupled mode, consisting of both a symmetric and anti-symmetric fluid sloshing mode and a vessel mode, with all three in phase $\left(\mu_{B}=\mu_{A}\right)$ or out of phase with $\mu_{B}-\mu_{A}=\pi$. In this case the vessel oscillates as $B \neq 0$ but the motion would also include a symmetric sloshing mode. As the symmetric mode exerts no resultant force on the vessel, the amplitude of the oscillation would depend solely on the value of $B$. An example of the free-surface elevation with $\mu_{A}=\mu_{B}$ is given in figure 4 .

$$
\text { 6.4. } A \neq 0, B \neq 0 ; \text { fully coupled with } \mu_{B}=\mu_{A}+\frac{1}{2} \pi \text { or } \mu_{B}=\mu_{A}+\frac{3}{2} \pi
$$

In this case the equations $(6.2)$ reduce to

$$
\left[\begin{array}{cc}
a_{2} & a_{3}-a_{4} \\
a_{3}-a_{4} & b_{2}
\end{array}\right]\left(\begin{array}{c}
r_{A}^{2} \\
r_{B}^{2}
\end{array}\right)=-\omega_{n, 2}\left(\begin{array}{l}
a_{1} \\
b_{1}
\end{array}\right) .
$$

This equation is uniquely solvable for $r_{A}^{2}$ and $r_{B}^{2}$ if

$$
a_{2} b_{2}-\left(a_{3}-a_{4}\right)^{2} \neq 0,
$$

and the fixed points exist if both $r_{A}^{2}$ and $r_{B}^{2}$ are positive. The stability exponent in this case is

$$
\lambda^{2}=-4 a_{4} r_{A}^{2} r_{B}^{2}\left(\frac{a_{2}}{a_{0}^{2}}+\frac{b_{2}}{b_{0}^{2}}-2 \frac{a_{3}-a_{4}}{a_{0} b_{0}}\right),
$$

and the energy value for this class is

$$
H_{A B}=-\frac{1}{2} \omega_{n, 2}^{2} \frac{b_{2} a_{1}^{2}+a_{2} b_{1}^{2}-2 a_{1} b_{1}\left(a_{3}-a_{4}\right)}{a_{2} b_{2}-\left(a_{3}-a_{4}\right)^{2}} .
$$

This solution is a fully coupled mode, consisting of both a symmetric and anti-symmetric fluid sloshing mode and a vessel mode, with the symmetric mode $\frac{1}{2} \pi$ or $\frac{3}{2} \pi$ out of phase from the vessel motion and anti-symmetric fluid mode. This case is similar to that in $\S 6.4$, except with a different free-surface elevation, for which an example when $\mu_{A}=\mu_{B}+\pi / 2$ is shown in figure 5 .

A sample of numerical values is given in Table 2. A range of other values of the coefficients of the normal form for different fluid heights $h_{0}$ can be found in the supplementary material.

\section{Heteroclinic connections and energy transfer}

It can be shown that all the orbits of the differential equations (1.2) lie on an spheroid (Cushman \& Rod 1982; Cotter 1986), thus any unstable fixed point must either connect to itself (homoclinic connection) or to another unstable fixed point (heteroclinic connection). The energy of the system is constant along any orbit, so two distinct equilibra can have a heteroclinic connection only if they have the same energy value.

The orbits on the spheroid can be seen explicitly via the following construction. Introduce new coordinates

$$
\begin{aligned}
& I_{1}=A^{*} B+A B^{*}, \\
& I_{2}=\mathrm{i}\left(A^{*} B-A B^{*}\right), \\
& I_{3}=a_{0}|A|^{2}-b_{0}|B|^{2}, \\
& I_{4}=a_{0}|A|^{2}+b_{0}|B|^{2},
\end{aligned}
$$

which satisfy

$$
a_{0} b_{0}\left(I_{1}^{2}+I_{2}^{2}\right)+I_{3}^{2}=I_{4}^{2} .
$$

For fixed $I_{4}>0$ this equation describes a spheroid in $\left(I_{1}, I_{2}, I_{3}\right)$ space since $a_{0}$ and $b_{0}$ are both positive (see Appendix B). 

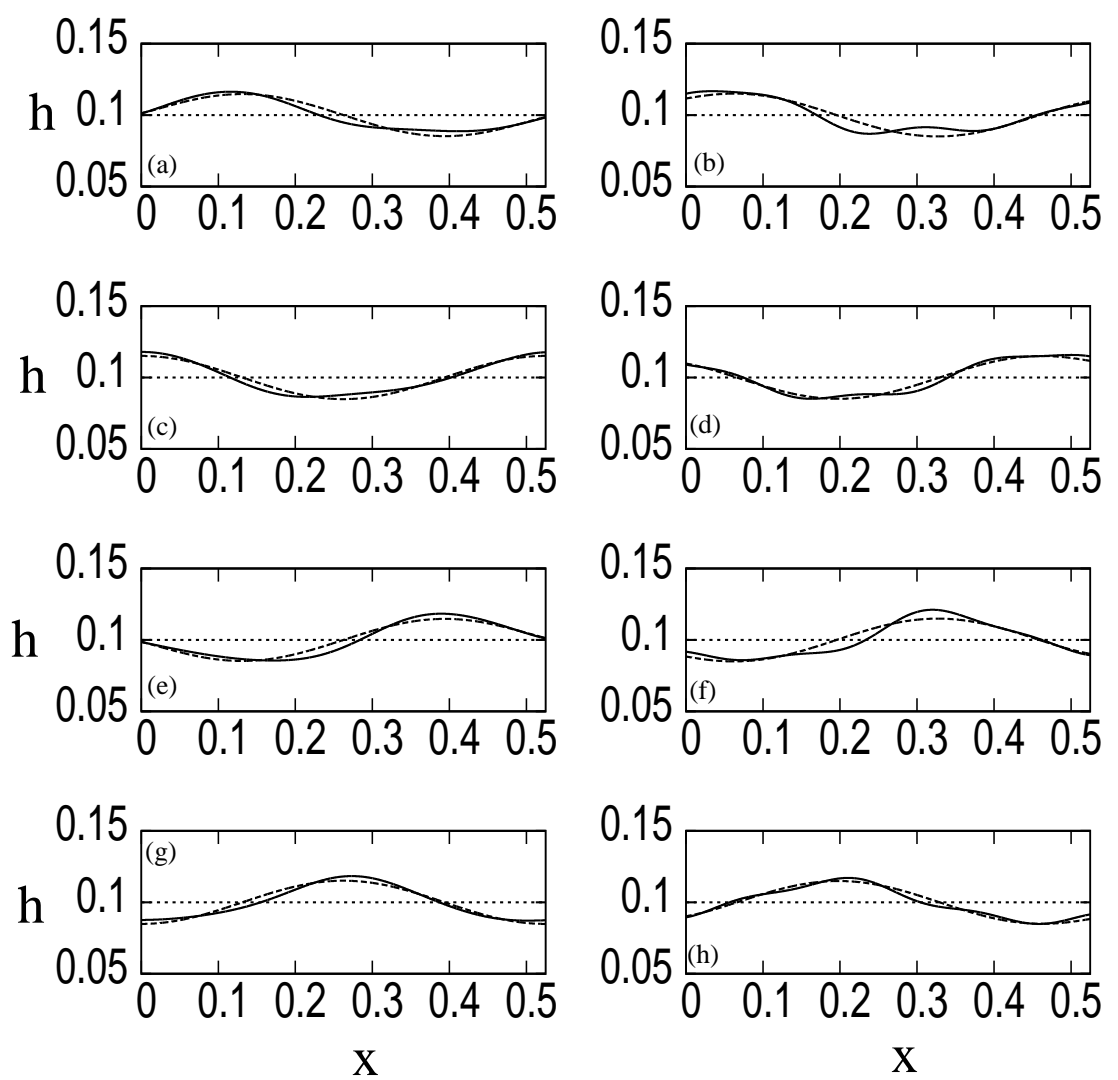

Figure 5. Plot of $h(x, t)$ for the conditions given in figure 4 except here $\widetilde{B}=0.01 e^{\mathrm{i} \pi / 2}$.

Differentiating the new coordinates with respect to $\tau$ and using (1.2) gives,

$$
\begin{aligned}
& \dot{I}_{1}=\gamma_{1} I_{2}+\left(\frac{a_{4}}{a_{0} b_{0}}+\gamma_{2}\right) I_{2} I_{3}, \\
& \dot{I}_{2}=-\gamma_{1} I_{1}+\left(\frac{a_{4}}{a_{0} b_{0}}-\gamma_{2}\right) I_{1} I_{3}, \\
& \dot{I}_{3}=-2 a_{4} I_{1} I_{2},
\end{aligned}
$$

where

$$
\gamma_{1}=\left(\frac{a_{1}}{a_{0}}-\frac{b_{1}}{b_{0}}\right)+\frac{1}{2}\left(\frac{a_{2}}{a_{0}^{2}}-\frac{b_{2}}{b_{0}^{2}}\right) I_{4} \quad \text { and } \quad \gamma_{2}=\frac{1}{2}\left(\frac{a_{2}}{a_{0}^{2}}-\frac{2 a_{3}}{a_{0} b_{0}}+\frac{b_{2}}{b_{0}^{2}}\right) .
$$

The fourth coordinate satisfies

$$
\dot{I}_{4}=0 \text {. }
$$

For each fixed positive value of $I_{4}$, equation (7.4) combined with (7.2) assure that all trajectories of (7.3) lie on the spheroid (7.2). The governing equations (7.3) have a close similarity to the rigid body equations in mechanics, and are completely integrable. Indeed, expressions for the heteroclinic orbits can be explicitly constructed. See $§ 3$, and particularly equation (3.9), in Holmes \& Marsden (1983) where explicit expressions for the heteroclinic orbits for the rigid body are written down. Analogous solutions can be developed for (7.3). The heteroclinic orbits are solutions which tend to one pure mode as $t \rightarrow-\infty$ and to the other pure mode as $t \rightarrow+\infty$.

An example of the orbit structure that would exist if the pure modes (blue dots) were connected by a heteroclinic connection is shown in Figure 6. The equilibria correspond to periodic solutions in the physical problem, and the orbits connecting equilibria on the sphere correspond to pathways for energy transfer in the physical problem. Consider $|A|^{2}$ as a measure of the energy of anti-symmetric fluid mode coupled to the vessel and $|B|^{2}$ as a measure of the amplitude of the symmetric fluid mode. Then along a heteroclinic connection one energy is decreasing and one increasing. This is the sense in which energy is transferred between modes, and hence physical configurations. The position of the fixed points are found using the information in appendix D. 


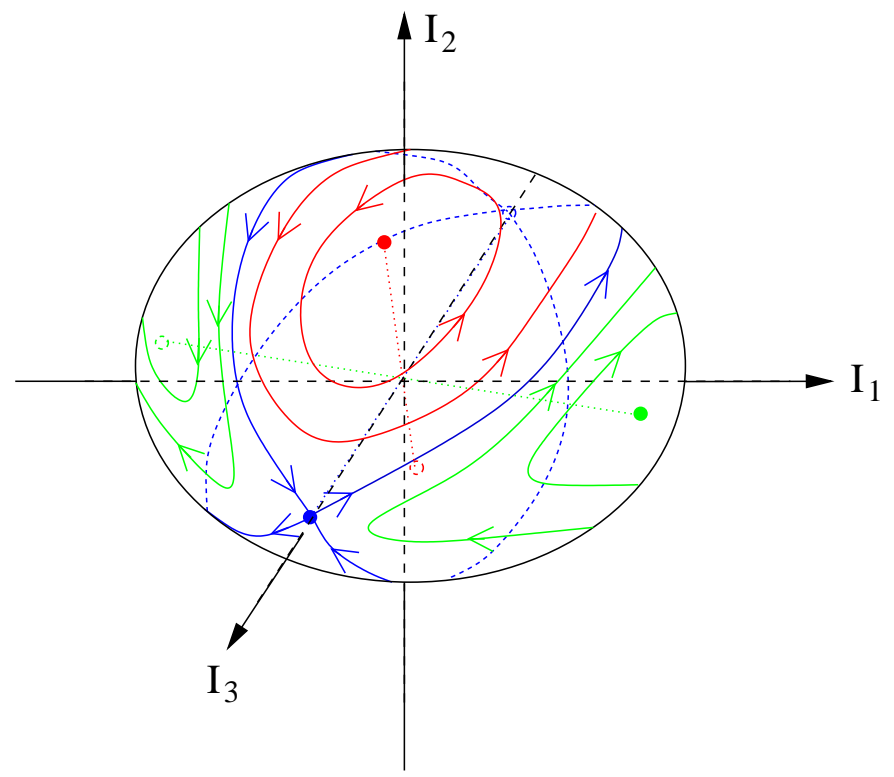

Figure 6. (colour online) Typical pattern of solutions of the normal form equations when the two pure modes (blue dots) are joined by a heteroclinic connection.

The analysis of the perturbed rigid body in Holmes \& Marsden (1983) also gives an indication of the implication of adding forcing to the coupled problem here near resonance. The theory in Holmes \& Marsden (1983) shows that periodic perturbation (such as periodic forcing) leads to chaotic solutions.

\section{Computing parameter values for connections}

Here we analyse the stability properties of the fixed points and determine parameter regimes where two fixed points are connected to one another via a heteroclinic connection, giving a pathway for energy transfer between the two modes.

In figure 7 we plot the quantities $\lambda^{2}$ and $H$ as a function of $h_{0}$ for the pure and mixed sloshing modes for $n=1$. Unstable fixed points occur when the quantity $\lambda^{2}>0$ and the fixed point is then a saddle point on the spheroid. Two fixed points can only have a heteroclinic connection to one another if both points exist, are unstable and both points have the same energy value $H$ for a particular value of $h_{0}$. Note that in panels (c) and (d) we only plot the mixed mode stability criteria and energy function if a solution exists. Heteroclinic orbits for mixed modes are common, because each result in figures $7(\mathrm{c})$ and $7(\mathrm{~d})$ correspond to a pair of mixed modes. Therefore, at $h=0.35 \mathrm{~m}$ for example, there exists a heteroclinic connection between a pair of mixed modes, one where the symmetric mode is in phase and one where it is out of phase with the vessel motion. However, heteroclinic connections between the mixed modes generally leads to less dramatic coupled motion than a heteroclinic connection between the two pure modes.

There exists three values of $h_{0} \in[0,0.4]$ where the energy of the two pure modes are equal, but only one such value of $h_{0}$ exists where both modes are simultaneously unstable and can both exist for the same value of $\omega_{n, 2}$. This occurs at $h_{0}=0.0805 \mathrm{~m}(\delta=0.1534)$. In figure $7(\mathrm{~b})$ the dashed line close to this value might be mistaken for an asymptote, but when this section of the figure is blown up in figure 8(b) it is clear that this is not the case. Here we see that the energy for the two pure sloshing modes is $H_{a}=H_{b}=0.1261 \omega_{1,2}^{2} \mathrm{kgm}^{2} \mathrm{~s}^{-2}$ at $h_{0}=0.0805 \mathrm{~m}$. Both these points exist simultaneously if $\omega_{1,2}>0$, which corresponds to the nonlinearity being a 'soft-spring' type nonlinearity. This shows that at this value of $h_{0}$ it is possible for the symmetric sloshing modes and the anti-symmetric sloshing modes (which are linked to the motion of the vessel) to transfer energy between one another. The most extreme cases of this energy transfer would be:

(a) A vessel moving back and forth coupled to the fluid sloshing with an anti-symmetric mode transfers all its energy to a symmetric fluid mode and the vessel comes to rest, or

(b) A stationary vessel containing only a symmetric sloshing mode begins to swing as its energy is transfered to the anti-symmetric modes and hence the vessel. 
(a)
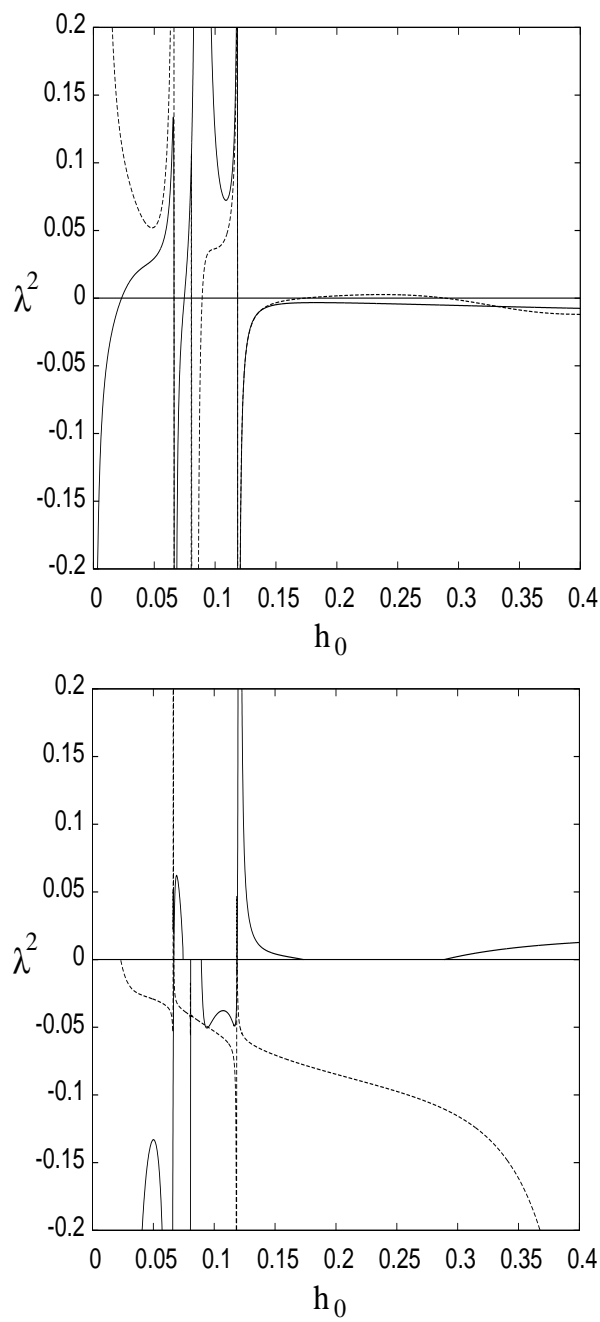

(b)
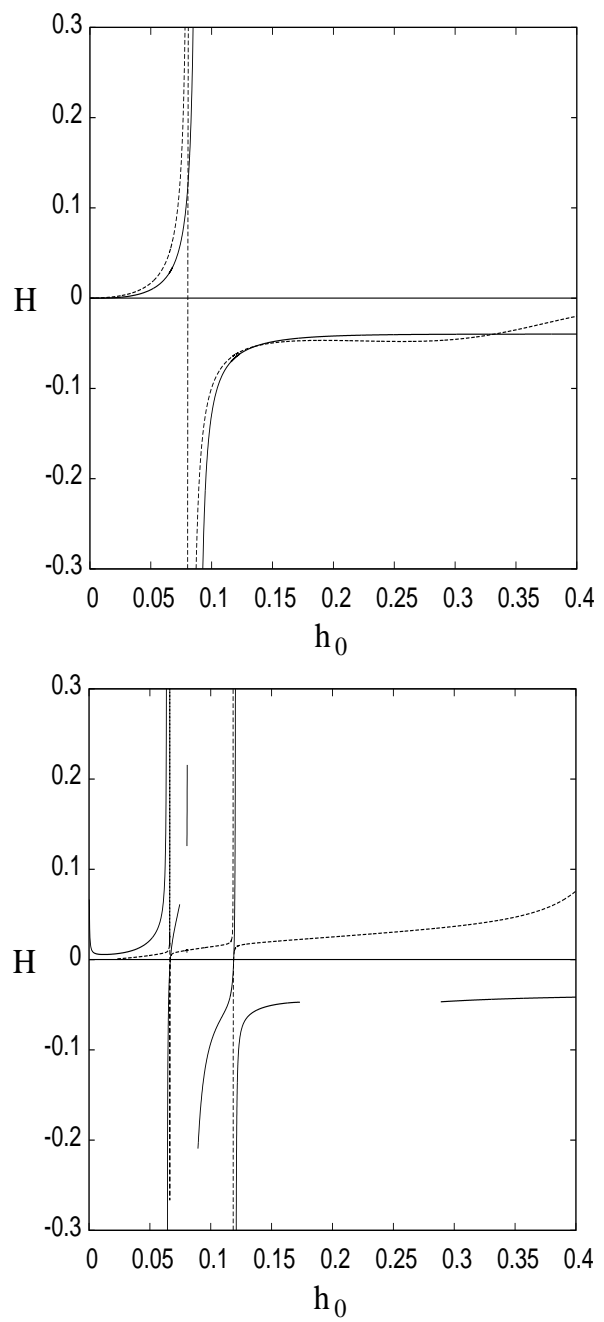

Figure 7. Plot of $\lambda^{2}$ and $H$ as a function of $h_{0}$ for $n=1$. Panels (a) and (b) give the pure $A$ modes (solid line) and pure $B$ modes (dashed line) while panels (c) and (d) give the mixed modes in $\S 6.3$ (solid line) and those described in $\S 6.4$ (dashed line). Note in panels (c) and (d) that the value of $\lambda^{2}$ and $H$ are only plotted if both $r_{A}$ and $r_{B}$ are real, and thus the solution exists.

If we consider a fluid height close to this heteroclinic connection (see table 2) then we observe the energy passing back and forth between the pure modes. This is seen in figure 9 . Here we have chosen $\epsilon=0.01$ for illustration purposes with $\omega_{1,2}=1 \mathrm{~s}^{-1}, A=1 \mathrm{kgm}^{2} \mathrm{~s}^{-2}$ and $B=10^{-10}$ initially. The constant $B$ has to be non-zero initially to allow the energy to pass to the anti-symmetric modes, if it is set identically to zero then it will remain zero for all times. The normal form equations (1.2) are then integrated to give the time variation of $A$ and $B$. Figure $9(\mathrm{~b})$ shows the vessel is stationary initially and then begins to oscillate once the anti-symmetric modes have the energy of the symmetric mode transfered to them (see panel (a)) and then the amplitude of the oscillations decrease and the vessel comes to rest again as the energy is transfered back to the symmetric modes. The resonance for $n=1$ with $h_{0}=0.0805 \mathrm{~m}$ occurs when $l=1.2077 \mathrm{~m}$ which is within the range accessible by experiments and so should be observable.

The nonlinear energy transfer also occurs for higher frequency sloshing modes for a particular value of $h_{0}$. Figure 10 shows that for $n=2$ a heteroclinic connection between the two pure modes exists when $h_{0}=0.0402 \mathrm{~m}$ and $H_{A}=H_{B}=0.0155 \omega_{2,2}^{2} \mathrm{kgm}^{2} \mathrm{~s}^{-2}$. In this case the 1:1 resonance occurs for a string length of $l=0.3334 \mathrm{~m}$, which is again in the parameter range accessible in experiments. As for the $n=1$ case, $\omega_{2,2}$ is again restricted to positive values. This energy transfer also occurs for $n>2$, but in these cases, parameters such as the string length and fluid height would correspond to values which would be difficult to replicate in experiments, so they are not considered in more detail here. 

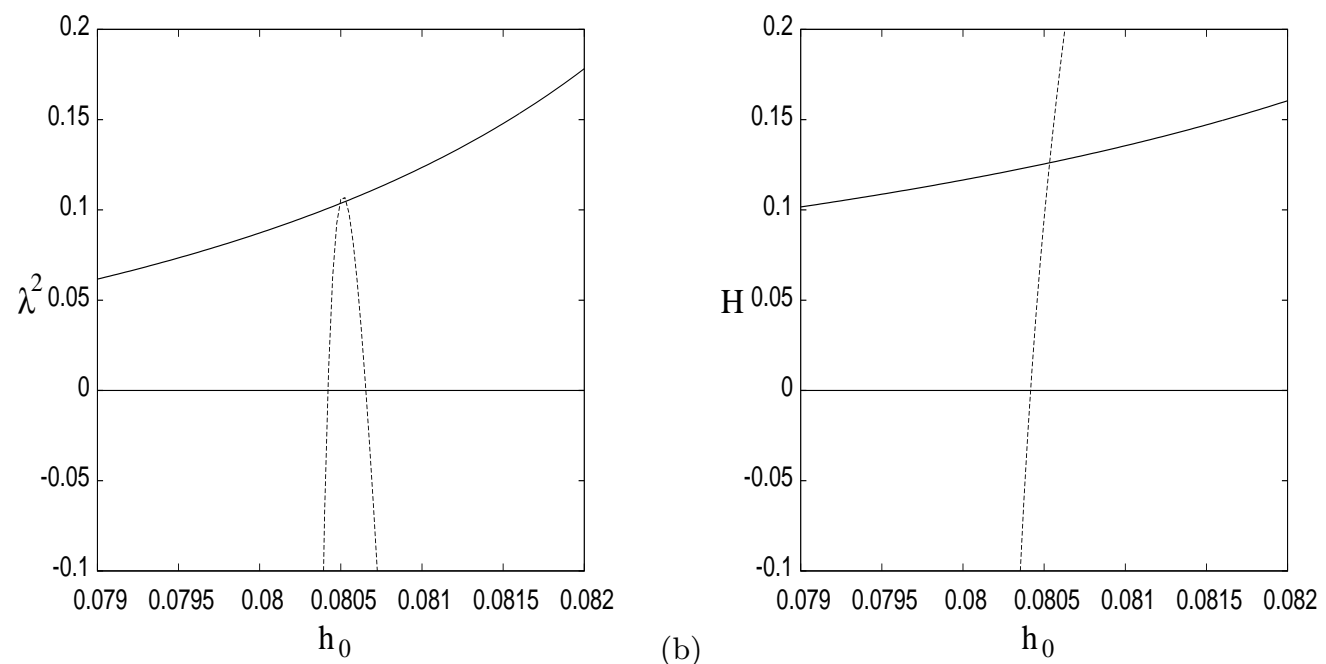

FiguRE 8. Blow up of figures 7 (a) and (b).

\begin{tabular}{cr}
\hline \hline Parameter & Value \\
\hline \hline & \\
$h_{0}$ & 0.0805325 \\
$\delta$ & 0.1533952 \\
$a_{0}$ & 152.3300743 \\
$a_{1}$ & 16.2771983 \\
$a_{2}$ & -1050.6922918 \\
$a_{3}$ & -21675.4703815 \\
$a_{4}$ & 20615.1349791 \\
$b_{0}$ & 256.8118394 \\
$b_{1}$ & 27.4415756 \\
$b_{2}$ & -3001.5860613 \\
& \\
\hline \hline
\end{tabular}

TABLE 2. Values of the normal form parameters close to the heteroclinic connection of the pure sloshing modes with $n=1$.

\section{Conclusions}

This paper determined the nonlinear solution to the coupled sloshing and vessel motion problem in a suspended rectangular tank. A solution to the nonlinear coupled equations was found in the form of an asymptotic expansion in increasing powers of $\epsilon \ll 1$ which was a measure of the disturbance magnitude from the quiescent solution. Alternatively $\epsilon$ can be defined using averaging. If $h_{1}$ is orthogonal to all higher $h_{j}$, then

$$
\epsilon:=\frac{\overline{\left(h-h_{0}\right) h_{1}}}{\overline{h_{1}^{2}}},
$$

where the bar denotes an average over $(x, t, \tau)$. Results for the evolution of the free-surface profile were presented up to $O\left(\epsilon^{2}\right)$ and were found to contain higher spatial harmonics in the free surface profile.

The nonlinear normal form of the solution was constructed close to the internal 1:1 resonance where the symmetric fluid modes couple to the anti-symmetric fluid modes and the vessel motion. In this solution it was discovered that for a single fluid depth $h_{0}$ (for each symmetric mode of wavenumber $n$ ) there exists a heteroclinic connection between the pure symmetric sloshing modes, and the pure antisymmetric sloshing modes. This heteroclinic connection is a pathway for energy transfer between these pure modes. The anti-symmetric modes couple to the motion of the vessel, thus energy transfer between these modes can lead to a dramatic vessel motion. The most extreme case is when an oscillating vessel 

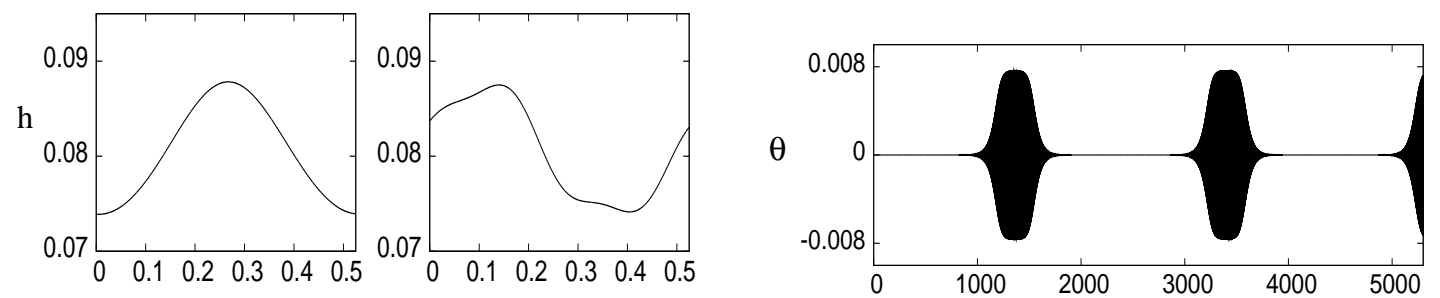

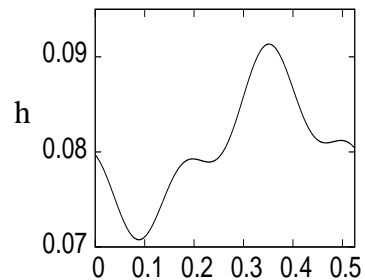

(a)

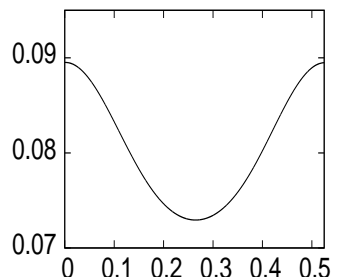

$\mathrm{L}$

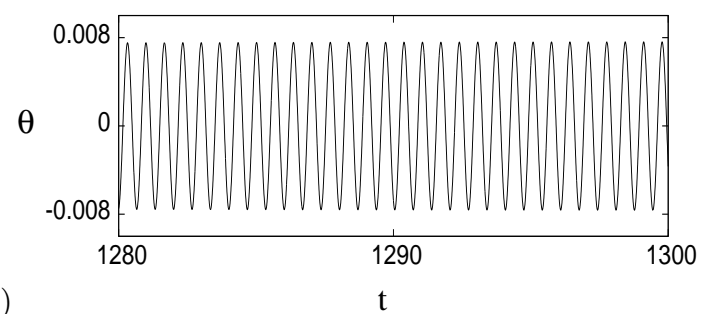

Figure 9. Plot of (a) $h(x, t)$ and (b) $\theta(t)$ for the parameter regime close to the heteroclinic connection given in table 2 with $n=1$. The time evolution of the functions $A$ and $B$ are found by integrating (1.2) with $\epsilon=0.01$, $\omega_{1,2}=1 \mathrm{~s}^{-1}$ and initial values of $A=1 \mathrm{~m}^{2} \mathrm{~s}^{-1}$ and $B=10^{-10}$. The corresponding free-surface profiles are then found using (5.2). Here $t$ is the original unscaled time from $(4.1)=\widetilde{t} / \omega$. In panel (a) the free-surfaces are plotted at $t=1069,1282,1496$ and 1710 from top left to bottom right. The fast time oscillations are highlighted in lower figure of panel (b).

(a)

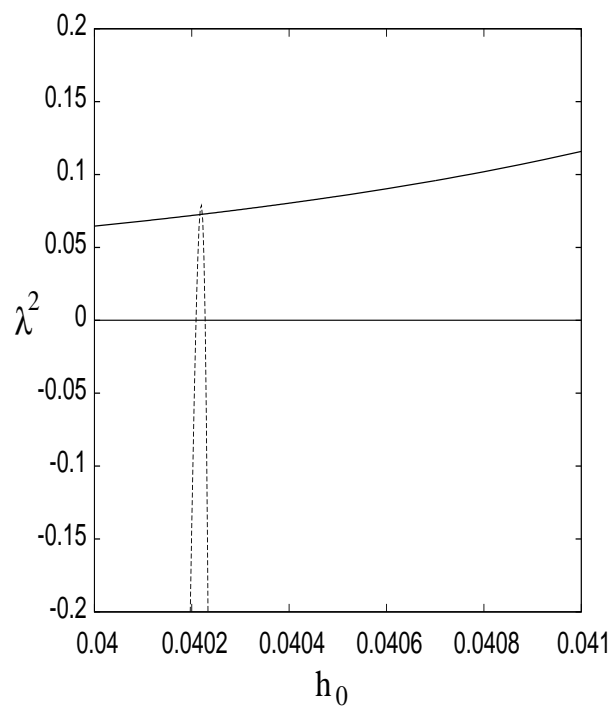

(b)

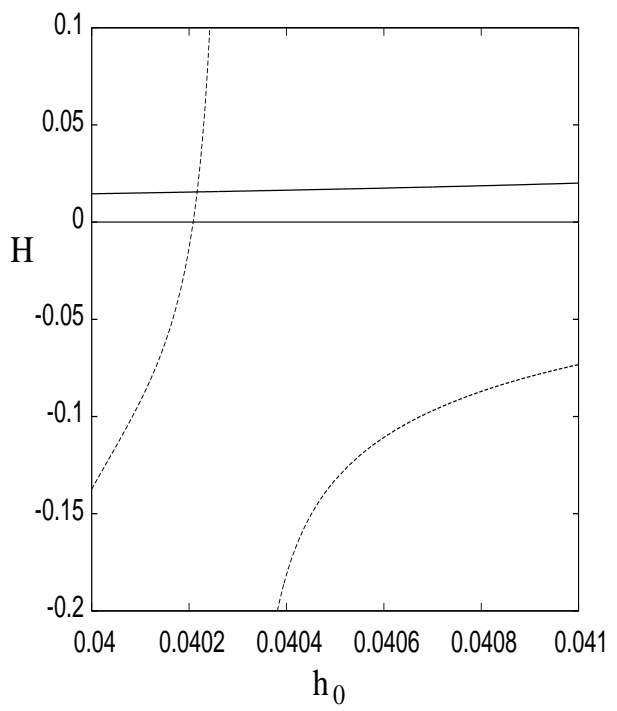

Figure 10. Close up of $\lambda^{2}$ and $H$ for the pure sloshing modes with $n=2$ close to critical value of $h_{0}$. The line styles are as in figure 7 .

containing only anti-symmetric modes comes to rest as the energy is transfered to the symmetric modes, or vice versa.

While this mechanism has been demonstrated for a particular example, there are general features that will be present in any system with vehicle motion coupled to a sloshing fluid. A key feature was a $1: 1$ resonance between a fluid mode and the vessel mode, and this resonance will be generally present in a physical configuration. Once such a resonance is identified it would be sensible to adjust system parameters to avoid the uncontrolled energy transfer. On the other hand, the converse situation might be advantageous: use the resonance to extract energy from the vessel motion and bring it to rest.

\section{Appendix A. Solution of the second order nonlinear equations}

Here we determine the solution for $\hat{\phi}_{2}(x, y, \tau)$ and $\hat{\theta}_{2}(\tau)$ from the system of equations (4.2)-(4.8). 
Firstly, by writing

$$
\hat{\phi}_{2}=\bar{\phi}_{2}-\frac{1}{4 \omega_{n}^{2}} F+\frac{1}{2} l \omega_{n} B^{2}\left(y-h_{0}\right),
$$

where

$$
\begin{aligned}
F= & \frac{1}{4} \omega_{n} A^{2} \beta_{n}^{2}\left(1+3 \tanh ^{2} \beta_{n} h_{0}\right)+B^{2} l^{2} \omega_{n}^{5} \sum_{m=0}^{\infty} \frac{1}{L \sigma_{m, n}}\left[\frac{8 \omega_{n}^{2}}{L \alpha_{m}^{2} \sigma_{m, n}}+\frac{12 \omega_{n}^{2}}{L \alpha_{m}^{2} \sigma_{m, n}} \tanh ^{2} \alpha_{m} h_{0}\right. \\
& \left.+p_{m}-\frac{4 \omega_{n}^{2}}{L \alpha_{m}^{2} \sigma_{m, n}}\right]-\frac{1}{2} l^{2} \omega_{n}^{3} B^{2}-8 l^{2} \omega_{n}^{5} B^{2} \sum_{m=0}^{\infty} \frac{1}{L^{2} \alpha_{m}^{2} \sigma_{m, n}},
\end{aligned}
$$

is a constant, the system of equations reduce to

$$
\begin{array}{rll}
\bar{\phi}_{2 x x}+\bar{\phi}_{2 y y}=0 & \text { in } \quad & 0<y<h_{0}, 0<x<L, \\
4 \omega_{n}^{2} \bar{\phi}_{2}+g \bar{\phi}_{2 y}=f(x) & \text { on } \quad y=h_{0}, \\
\bar{\phi}_{2 y}=0 & \text { on } \quad y=0, \\
\bar{\phi}_{2 x}=-2 \omega_{n} l \hat{\theta}_{2} & \text { on } \quad & x=0, L,
\end{array}
$$

for the fluid motion and

$$
-4 \omega_{n}^{2} m_{v} \hat{\theta}_{2}+\frac{g}{l}\left(m_{v}+m_{f}\right) \hat{\theta}_{2}=-\frac{2 \omega_{n} W}{l} \int_{0}^{L} \int_{0}^{h_{0}} \rho \bar{\phi}_{2 x} d y d x-\frac{\omega_{n}^{2} W}{g l} \int_{0}^{L} \rho \hat{\phi}_{1} \hat{\phi}_{1 x} d x,
$$

for the vessel. Here

$$
\begin{aligned}
& f(x)=\frac{1}{2} \omega_{n} \sum_{r=1}^{\infty}\left[\frac{3}{2} A^{2} \beta_{n}^{2} \delta_{r, 2 n}\left(\tanh ^{2} \beta_{n} h_{0}-1\right)+\frac{2 \omega_{n}^{4}}{L \sigma_{r, n}} l^{2} B^{2} \delta_{r}\left\{-\frac{32 \omega_{n}^{2}}{L \beta_{r}^{2} \sigma_{r, n}}\right.\right. \\
& \left.+\frac{48 \omega_{n}^{2}}{L \beta_{r}^{2} \sigma_{r, n}} \tanh ^{2} \frac{1}{2} \beta_{r} h_{0}+p_{r}-\frac{16 \omega_{n}^{2}}{L \beta_{r}^{2} \sigma_{r, n}}\right\}-16 l^{2} \omega_{n}^{4} B^{2} \sum_{m=0}^{\infty} \frac{1}{L^{2} \sigma_{m, n}\left(\beta_{r}^{2}-\alpha_{m}^{2}\right)} \\
& +\frac{1}{2} l^{2} \omega_{n}^{2} B^{2} \sum_{\substack{m=0 \\
q=m+r}}^{\infty}\left\{\frac{64 \omega_{n}^{4}}{L^{2} \alpha_{m} \alpha_{q} \sigma_{m, n} \sigma_{q, n}}+\frac{96 \omega_{n}^{4}}{L^{2} \alpha_{m} \alpha_{q} \sigma_{m, n} \sigma_{q, n}} \tanh \alpha_{m} h_{0} \tanh \alpha_{q} h_{0}\right. \\
& \left.+\left(p_{m}-\frac{4 \omega_{n}^{2}}{L \alpha_{m}^{2} \sigma_{m, n}}\right)\left(\frac{4 \omega_{n}^{2}}{L \sigma_{q, n}}\right)+\left(\frac{4 \omega_{n}^{2}}{L \sigma_{m, n}}\right)\left(p_{q}-\frac{4 \omega_{n}^{2}}{L \alpha_{q}^{2} \sigma_{q, n}}\right)\right\} \\
& +\frac{1}{2} l^{2} \omega_{n}^{2} B^{2} \sum_{\substack{m=0 \\
q=r-1-m \\
q>m}}^{\infty}\left\{-\frac{64 \omega_{n}^{4}}{L^{2} \alpha_{m} \alpha_{q} \sigma_{m, n} \sigma_{q, n}}+\frac{96 \omega_{n}^{4}}{L^{2} \alpha_{m} \alpha_{q} \sigma_{m, n} \sigma_{q, n}} \tanh \alpha_{m} h_{0} \tanh \alpha_{q} h_{0}\right. \\
& \left.\left.+\left(p_{m}-\frac{4 \omega_{n}^{2}}{L \alpha_{m}^{2} \sigma_{m, n}}\right)\left(\frac{4 \omega_{n}^{2}}{L \sigma_{q, n}}\right)+\left(\frac{4 \omega_{n}^{2}}{L \sigma_{m, n}}\right)\left(p_{q}-\frac{4 \omega_{n}^{2}}{L \alpha_{q}^{2} \sigma_{q, n}}\right)\right\}\right] \cos \beta_{r} x \\
& +\frac{1}{4} l \omega_{n}^{2} A B \sum_{r=0}^{\infty}\left[\sum _ { \substack { q = n + r \\
q = n - r - 1 } } \left\{-\frac{16 \beta_{n} \omega_{n}^{2}}{L \alpha_{q} \sigma_{q, n}}-\frac{24 \beta_{n} \omega_{n}^{2}}{L \alpha_{q} \sigma_{q, n}} \tanh \beta_{n} h_{0} \tanh \alpha_{q} h_{0}\right.\right. \\
& \left.+\frac{4 \omega_{n}^{2}}{L \sigma_{q, n}}-\beta_{n}^{2}\left(p_{q}-\frac{4 \omega_{n}^{2}}{L \alpha_{q}^{2} \sigma_{q, n}}\right)\right\}+\sum_{\substack{q=r-n \\
q>0}}\left\{\frac{16 \beta_{n} \omega_{n}^{2}}{L \alpha_{q} \sigma_{q, n}}-\frac{24 \beta_{n} \omega_{n}^{2}}{L \alpha_{q} \sigma_{q, n}} \tanh \beta_{n} h_{0} \tanh \alpha_{q} h_{0}\right. \\
& \left.\left.+\frac{4 \omega_{n}^{2}}{L \sigma_{q, n}}-\beta_{n}^{2}\left(p_{q}-\frac{4 \omega_{n}^{2}}{L \alpha_{q}^{2} \sigma_{q, n}}\right)\right\}-\left(\frac{8 \beta_{n}^{2}}{L\left(\beta_{n}^{2}-\alpha_{r}^{2}\right)}\right)\right] \cos \alpha_{r} x \\
& =\sum_{r=1}^{\infty}\left(A^{2} d_{r}^{A}+B^{2} d_{r}^{B}\right) \cos \beta_{r} x+\sum_{r=0}^{\infty} A B d_{r}^{A B} \cos \alpha_{r} x,
\end{aligned}
$$

and the constants $p_{r}=-4 /\left(\alpha_{r}^{2} L\right)$ come from expanding $x-L / 2$ as an infinite cosine expansion

$$
x-\frac{1}{2} L=\sum_{r=0}^{\infty} p_{r} \cos \alpha_{r} x,
$$


which is valid for $x \in[0, L]$. The constants $\delta_{r, 2 n}$ and $\delta_{r}$ appearing in (A 6$)$ are defined as

$$
\delta_{r, 2 n}=\left\{\begin{array}{cc}
1 & r=2 n \\
0 & \text { otherwise }
\end{array}, \quad \delta_{r}=\left\{\begin{array}{ll}
1 & \text { for } r \text { odd } \\
0 & \text { otherwise }
\end{array} .\right.\right.
$$

The system of equations (A 1 ) and (A 5) can now be solved by splitting $\bar{\phi}_{2}$ into a part proportional to $\hat{\theta}_{2}$ and a part not proportional to $\hat{\theta}_{2}$

$$
\bar{\phi}_{2}=G_{1}(x, y, \tau)+\hat{\theta}_{2}(\tau)\left(-2 \omega_{n} l\left(x-\frac{1}{2} L\right)+G_{2}(x, y)\right) .
$$

The functions $G_{1}$ and $G_{2}$ both satisfy

$$
\begin{aligned}
G_{x x}+G_{y y}=0 & \text { in } 0<y<h_{0}, 0<x<L, \\
-4 \omega_{n}^{2} G+g G_{y}=\chi_{i}(x) & \text { on } \quad y=h_{0}, \\
G_{y}=0 & \text { on } \quad y=0, \\
G_{x}=0 & \text { on } \quad x=0, L,
\end{aligned}
$$

where $\chi_{1}(x)=f(x)$ in the equation for $G_{1}(x, y, \tau)$ and $\chi_{2}(x)=-8 \omega_{n}^{3} l\left(x-\frac{1}{2} L\right)$ in the equation for $G_{2}(x, y)$.

As $\chi_{2}(x)$ can be solely expanded in terms of odd cosine functions (see (A 7)), the general solution to the above system of equations satisfying all the equations except the free-surface boundary condition is

$$
G_{2}(x, y)=\sum_{r=0}^{\infty} a_{r} \frac{\cosh \left(\alpha_{r} y\right)}{\cosh \left(\alpha_{r} h_{0}\right)} \cos \left(\alpha_{r} x\right)
$$

When this is substituted into the free-surface condition we obtain the value of the constants as

$$
a_{r}=\frac{24 \omega_{n}^{3} l}{\alpha_{r}^{2} L\left(g \alpha_{r} \tanh \alpha_{r} h_{0}-4 \omega_{n}^{2}\right)} .
$$

A similar solution exists for $G_{1}$, but in this case $\chi_{1}(x)$ contains a sum of both even and odd cosine functions, so here the solution satisfying all the equations except the free-surface condition is

$$
G_{1}(x, y, \tau)=\sum_{r=1}^{\infty} b_{r} \frac{\cosh \left(\beta_{r} y\right)}{\cosh \left(\beta_{r} h_{0}\right)} \cos \left(\beta_{r} x\right)+\sum_{r=0}^{\infty} c_{r} \frac{\cosh \left(\alpha_{r} y\right)}{\cosh \left(\alpha_{r} h_{0}\right)} \cos \left(\alpha_{r} x\right) .
$$

When this is substituted into the free-surface condition the constants are found to be

$$
b_{r}=\frac{A^{2} d_{r}^{A}+B^{2} d_{r}^{B}}{g \beta_{r} \tanh \beta_{r} h_{0}-4 \omega_{n}^{2}}, \quad c_{r}=\frac{A B d_{r}^{A B}}{g \alpha_{r} \tanh \alpha_{r} h_{0}-4 \omega_{n}^{2}} .
$$

When these solutions are substituted back into $\hat{\phi}_{2}$ and subsequently substituted into the coupling equation (A 5), then value of $\hat{\theta}_{2}$ is found to be

$$
\hat{\theta}_{2}=\frac{4 \omega_{n} \rho W}{l \Lambda} \sum_{r=0}^{\infty} \frac{c_{r}}{\alpha_{r}} \tanh \alpha_{r} h_{0}-\frac{2 A B \omega_{n}^{3} \rho W}{g \Lambda}\left(\frac{1}{2} L+\sum_{r=0}^{\infty} \frac{4 \omega_{n}^{2}}{L \alpha_{r}^{2} \sigma_{r, n}}\right),
$$

where

$$
\Lambda=\left(m_{v}+m_{f}\right)\left(-4 \omega_{n}^{2}+\frac{g}{l}\right)-\frac{4 \omega_{n} \rho W}{l} \sum_{r=0}^{\infty} \frac{a_{r}}{\alpha_{r}} \tanh \alpha_{r} h_{0} .
$$


Therefore the forms of the $\zeta_{i}$ 's, $Z_{i}$ 's and $\Theta_{1}$ in (4.10) are

$$
\begin{aligned}
\zeta_{0}= & \sum_{r=1}^{\infty} \frac{d_{r}^{A}}{g \beta_{r} \tanh \beta_{r} h_{0}-4 \omega_{n}^{2}} \frac{\cosh \left(\beta_{r} y\right)}{\cosh \left(\beta_{r} h_{0}\right)} \cos \left(\beta_{r} x\right) \\
\zeta_{1}= & \sum_{r=0}^{\infty} \frac{d_{r}^{A B}}{g \alpha_{r} \tanh \alpha_{r} h_{0}-4 \omega_{n}^{2}} \frac{\cosh \left(\alpha_{r} y\right)}{\cosh \left(\alpha_{r} h_{0}\right)} \cos \left(\alpha_{r} x\right) \\
\zeta_{2}= & \sum_{r=1}^{\infty} \frac{d_{r}^{B}}{g \beta_{r} \tanh \beta_{r} h_{0}-4 \omega_{n}^{2}} \frac{\cosh \left(\beta_{r} y\right)}{\cosh \left(\beta_{r} h_{0}\right)} \cos \left(\beta_{r} x\right) \\
Z_{1}= & \frac{1}{2} l \omega_{n}, \\
Z_{2}= & -\frac{1}{2} l \omega_{n} h_{0}-\frac{1}{4} l^{2} \omega_{n}^{3} \sum_{m=0}^{\infty} \frac{1}{L \sigma_{m, n}}\left[\frac{8 \omega_{n}^{2}}{L \alpha_{m}^{2} \sigma_{m, n}}+\frac{12 \omega_{n}^{2}}{L \alpha_{m}^{2} \sigma_{m, n}} \tanh ^{2} \alpha_{m} h_{0}\right. \\
& \left.+p_{m}-\frac{4 \omega_{n}^{2}}{L \alpha_{m}^{2} \sigma_{m, n}}\right]+\frac{1}{8} l^{2} \omega_{n}+2 l^{2} \omega_{n}^{3} B^{2} \sum_{m=0}^{\infty} \frac{1}{L^{2} \alpha_{m}^{2} \sigma_{m, n}}, \\
Z_{3}= & -\frac{1}{16 \omega_{n}} \beta_{n}^{2}\left(1+3 \tanh ^{2} \beta_{n} h_{0}\right), \\
\Theta_{1}= & \frac{4 \omega_{n} \rho W}{l \Lambda} \sum_{r=0}^{\infty} \frac{d_{r}^{A B}}{\left(g \alpha_{r} \tanh _{r} h_{0}-4 \omega_{n}^{2}\right) \alpha_{r}} \tanh \alpha_{r} h_{0}-\frac{2 \omega_{n}^{3} \rho W}{g \Lambda}\left(\frac{1}{2} L+\sum_{r=0}^{\infty} \frac{4 \omega_{n}^{2}}{L \alpha_{r}^{2} \sigma_{r, n}}\right)
\end{aligned}
$$

\section{Appendix B. Coefficients of the Lagrangian}

The coefficients of the Lagrangian (5.7) are given below. We note that constants which contain a single $x$ integral of a function of $(x, y)$ are evaluated at $y=h_{0}$. The coefficients of the linear terms are

$$
\begin{aligned}
& a_{0}=\frac{\omega_{n}^{2} \rho W}{2 g} \int_{0}^{L} S_{0}^{2} d x=\frac{\omega_{n}^{2} m_{f}}{4 g h_{0}}, \\
& b_{0}=\frac{\omega_{n}^{2} \rho W}{2 g} \int_{0}^{L} S_{1}^{2} d x+\frac{l g}{2}\left(m_{v}+m_{f}\right), \\
& a_{1}=\frac{\omega_{n} \rho W}{2 g} \int_{0}^{L} S_{0}^{2} d x=\frac{\omega_{n} m_{f}}{4 g h_{0}}, \\
& b_{1}=\frac{\omega_{n} \rho W}{2 g} \int_{0}^{L} S_{1}^{2} d x+\frac{l g}{2 \omega_{n}}\left(m_{v}+m_{f}\right) .
\end{aligned}
$$


The coefficients of the nonlinear terms for the pure modes are

$$
\begin{aligned}
a_{2}= & -\frac{\rho W}{2} \int_{0}^{L} \int_{0}^{h_{0}}\left(\zeta_{0 x}^{2}+\zeta_{0 y}^{2}\right) d y d x+\frac{\rho W}{32 g^{3}} \int_{0}^{L}\left(-2 \omega_{n}^{4} S_{0}^{2} S_{0 x}^{2}+3 g^{2} S_{0 x}^{4}+11 \frac{\omega_{n}^{8}}{g^{2}} S_{0}^{4}\right. \\
& +48 \omega_{n}^{5} S_{0}^{2} Z_{3}+64 g^{2} \omega_{n}^{2} Z_{3}^{2}+16 g^{2} \omega_{n} S_{0 x}^{2} Z_{3}+128 g^{2} \omega_{n}^{2} Z_{3} \zeta_{0}+48 \omega_{n}^{5} S_{0}^{2} \zeta_{0} \\
& \left.-16 g^{2} \omega_{n} S_{0} S_{0 x} \zeta_{0 x}+64 g^{2} \omega_{n}^{2} \zeta_{0}^{2}+16 g^{2} \omega_{n} S_{0 x}^{2} \zeta_{0}-8 g^{2} C_{0}^{2}\right) d x \\
= & \frac{m_{f} \beta_{n}^{4}}{256 g h_{0}}\left(-\frac{9}{T_{n}^{2}}+16-5 T_{n}^{2}+6 T_{n}^{4}\right) \\
b_{2}= & -\frac{\rho W}{8} \int_{0}^{L} \int_{0}^{h_{0}}\left(4 \zeta_{2 x}^{2}+4 \zeta_{2 y}^{2}-4 l \omega_{n} \zeta_{2 y}+8 Z_{1} \zeta_{2 y}+l \omega_{n} S_{1 x}+4 Z_{1}^{2}-4 l \omega_{n} Z_{1}\right) d y d x \\
& +\frac{\rho W}{32 g^{3}} \int_{0}^{L}\left(-2 \omega_{n}^{4} S_{1}^{2} S_{1 x}^{2}+11 \frac{\omega_{n}^{8}}{g^{2}} S_{1}^{4}+3 g^{2} S_{1 x}^{4}-12 g^{2} \omega_{n} l S_{1 x}^{3}+12 g^{2} \omega_{n}^{2} l^{2} S_{1 x}^{2}+8 g \omega_{n}^{4} l S_{1}^{2}\right. \\
& +64 g^{2} \omega_{n}^{2} \zeta_{2}^{2}+16 g^{2} \omega_{n} S_{1 x}^{2} \zeta_{2}+48 \omega_{n}^{5} S_{1}^{2} \zeta_{2}-16 g^{2} \omega_{n} S_{1} S_{1 x} \zeta_{2 x}+16 g^{2} \omega_{n}^{2} l S_{1} \zeta_{2 x} \\
& -32 g^{2} \omega_{n}^{2} l S_{1 x} \zeta_{2}+128 g^{2} \omega_{n}^{2} Z_{1} h_{0} \zeta_{2}+64 g^{2} \omega_{n}^{2} Z_{2}^{2}-64 g^{2} \omega_{n}^{2} Z_{1}^{2} h_{0}^{2}+16 g^{2} \omega_{n} S_{1 x}^{2} Z_{2} \\
& +48 \omega_{n}^{5} S_{1}^{2} Z_{2}+128 g^{2} \omega_{n}^{2} Z_{2} \zeta_{2}+48 \omega_{n}^{5} Z_{1} h_{0} S_{1}^{2}-32 g^{2} \omega_{n}^{2} l S_{1 x} Z_{1} h_{0}-32 g^{2} \omega_{n}^{2} l S_{1 x} Z_{2} \\
& \left.+16 g^{2} \omega_{n} Z_{1} h_{0} S_{1 x}^{2}+128 g^{2} \omega_{n}^{2} Z_{1}^{2} h_{0}^{2}+128 g^{2} \omega_{n}^{2} Z_{1} h_{0} Z_{2}-8 g^{4} C_{3}^{2}\right) d x+\frac{l g}{64}\left(m_{v}+m_{f}\right),
\end{aligned}
$$

where $T_{n}=\tanh \left(\beta_{n} h_{0}\right)$ and $S_{0}(x, y)$ and $S_{1}(x, y)$ are defined in (3.12). The coefficients of the nonlinear terms for the mixed modes are

$$
\begin{aligned}
a_{3}= & -\frac{\rho W}{4} \int_{0}^{L} \int_{0}^{h_{0}}\left(\zeta_{1 x}^{2}+\zeta_{1 y}^{2}+4 l \omega_{n} \Theta_{1} \zeta_{1 x}\right) d y d x \\
& +\frac{\rho W}{16 g^{3}} \int_{0}^{L}\left(3 g^{2} S_{1 x}^{2} S_{0 x}^{2}-3 \omega_{n}^{4} S_{0}^{2} S_{1 x}^{2}-3 \omega_{n}^{4} S_{0 x}^{2} S_{1}^{2}+11 \frac{\omega_{n}^{8}}{g^{2}} S_{0}^{2} S_{1}^{2}+4 \omega_{n}^{4} S_{0} S_{0 x} S_{1} S_{1 x}\right. \\
& -6 g^{2} \omega_{n} l S_{0 x}^{2} S_{1 x}+4 \omega_{n}^{5} l S_{0}^{2} S_{1 x}-4 \omega_{n}^{5} l S_{0} S_{0 x} S_{1}+2 g^{2} \omega_{n}^{2} l^{2} S_{0 x}^{2}+24 \omega_{n}^{5} S_{0} S_{1} \zeta_{1}+8 g^{2} \omega_{n} S_{0 x} S_{1 x} \zeta_{1} \\
& -8 g^{2} \omega_{n}^{2} l S_{0 x} \zeta_{1}-4 g^{2} \omega_{n} S_{0 x} S_{1} \zeta_{1 x}-4 g^{2} \omega_{n} S_{0} S_{1 x} \zeta_{1 x}+4 g^{2} \omega_{n}^{2} l S_{0} \zeta_{1 x}+16 g^{2} \omega_{n}^{2} \zeta_{1}^{2} \\
& \left.-8 g^{2} \omega_{n}^{2} l \Theta_{1} S_{1} S_{0 x}-8 g^{2} \omega_{n}^{2} l \Theta_{1} S_{0} S_{1 x}-4 g^{4} C_{0} C_{3}\right) d x+\frac{l \Theta_{1}^{2}}{4}\left(g\left(m_{v}+m_{f}\right)-4 \omega_{n}^{2} m_{v} l\right), \\
a_{4}= & -\frac{\rho W}{2} \int_{0}^{L} \int_{0}^{h_{0}}\left(\zeta_{0 y} \zeta_{2 y}+\zeta_{0 x} \zeta_{2 x}\right) d y d x \\
& +\frac{\rho W}{32 g^{3}} \int_{0}^{L}\left(3 g^{2} S_{0 x}^{2} S_{1 x}^{2}+5 \omega_{n}^{4} S_{0}^{2} S_{1 x}^{2}+5 \omega_{n}^{4} S_{0 x}^{2} S_{1}^{2}+11 \frac{\omega_{n}^{8}}{g^{2}} S_{0}^{2} S_{1}^{2}-12 \omega_{n}^{4} S_{0} S_{0 x} S_{1} S_{1 x}\right. \\
& +2 g^{2} \omega_{n}^{2} l^{2} S_{0 x}^{2}+4 g \omega_{n}^{4} l S_{0}^{2}-6 g^{2} \omega_{n} l S_{0 x}^{2} S_{1 x}-8 \omega_{n}^{5} l S_{0}^{2} S_{1 x}+8 \omega_{n}^{5} l S_{0} S_{0 x} S_{1}+8 g^{2} \omega_{n} S_{1 x}^{2} \zeta_{0} \\
& +48 \omega_{n}^{5} S_{1}^{2} \zeta_{0}+8 g^{2} \omega_{n} S_{0 x}^{2} \zeta_{2}+48 \omega_{n}^{5} S_{0}^{2} \zeta_{2}+64 g^{2} \omega_{n}^{2} \zeta_{0} \zeta_{2}-8 g^{2} \omega_{n} S_{1} S_{1 x} \zeta_{0 x}-8 g^{2} \omega_{n} S_{0} S_{0 x} \zeta_{2 x} \\
& +8 g^{2} \omega_{n}^{2} l S_{1} \zeta_{0 x}-16 g^{2} \omega_{n}^{2} l S_{1 x} \zeta_{0}+8 g^{2} \omega_{n} S_{1 x}^{2} Z_{3}+48 \omega_{n}^{5} S_{1}^{2} Z_{3}+64 g^{2} \omega_{n}^{2} Z_{3} \zeta_{2}+8 g^{2} \omega_{n} Z_{2} S_{0 x}^{2} \\
& +48 \omega_{n}^{5} Z_{2} S_{0}^{2}+64 g^{2} \omega_{n}^{2} Z_{2} \zeta_{0}+64 g^{2} \omega_{n}^{2} Z_{1} Z_{3} h_{0}-16 g^{2} \omega_{n}^{2} l Z_{3} S_{1 x}+8 g^{2} \omega_{n} Z_{1} h_{0} S_{0 x}^{2}+48 \omega_{n}^{5} Z_{1} h_{0} S_{0}^{2} \\
& \left.+64 g^{2} \omega_{n}^{2} Z_{1} h_{0} \zeta_{0}+64 g^{2} \omega_{n}^{2} Z_{2} Z_{3}\right) d x . \\
&
\end{aligned}
$$

\section{Appendix C. Stability of the normal form equilibria}

In order to analyse the stability of the fixed points of the normal form equations (1.2), we first need to form four real equations for the components of the two complex unknowns $A$ and $B$. This can be achieved either by writing $A$ and $B$ in real and imaginary parts or by writing them in polar form. It turns out that that the method choice depends upon whether pure sloshing modes (i.e. $A \neq 0$ with $B=0$ or $A=0$ with $B \neq 0$ ) or mixed sloshing modes (both $A$ and $B$ are non-zero) are being considered.

$$
\text { C.1. Pure sloshing modes }(A \neq 0 \text { with } B=0 \text { or } A=0 \text { with } B \neq 0)
$$

For pure sloshing modes we write $A$ and $B$ in terms of real and imaginary parts

$$
A=u_{r}+i u_{i} \quad \text { and } \quad B=v_{r}+i v_{i},
$$


and the four real equations for the motion are

$$
\begin{aligned}
a_{0} \dot{u}_{r} & =\omega_{n, 2} a_{1} u_{i}+a_{2}\left(u_{r}^{2}+u_{i}^{2}\right) u_{i}+a_{3}\left(v_{r}^{2}+v_{i}^{2}\right) u_{i}+a_{4}\left(-\left(v_{r}^{2}-v_{i}^{2}\right) u_{i}+2 v_{r} v_{i} u_{r}\right), \\
-a_{0} \dot{u}_{i} & =\omega_{n, 2} a_{1} u_{r}+a_{2}\left(u_{r}^{2}+u_{i}^{2}\right) u_{r}+a_{3}\left(v_{r}^{2}+v_{i}^{2}\right) u_{r}+a_{4}\left(\left(v_{r}^{2}-v_{i}^{2}\right) u_{r}+2 v_{r} v_{i} u_{i}\right), \\
b_{0} \dot{v}_{r} & =\omega_{n, 2} b_{1} v_{i}+b_{2}\left(v_{r}^{2}+v_{i}^{2}\right) v_{i}+a_{3}\left(u_{r}^{2}+u_{i}^{2}\right) v_{i}+a_{4}\left(-\left(u_{r}^{2}-u_{i}^{2}\right) v_{i}+2 u_{r} u_{i} v_{r}\right), \\
-b_{0} \dot{v}_{i} & =\omega_{n, 2} b_{1} v_{r}+b_{2}\left(v_{r}^{2}+v_{i}^{2}\right) v_{r}+a_{3}\left(u_{r}^{2}+u_{i}^{2}\right) v_{r}+a_{4}\left(\left(u_{r}^{2}-u_{i}^{2}\right) v_{r}+2 u_{r} u_{i} v_{i}\right),
\end{aligned}
$$

where the dots denote differentiation with respect to $\tau$. The pure mode fixed points can be found at

$$
\begin{aligned}
& v_{r}=v_{i}=0 \quad \text { and } \quad \omega_{n, 2} a_{1}+a_{2}\left(u_{r}^{2}+u_{i}^{2}\right)=0, \quad(B=0) \text {, } \\
& u_{r}=u_{i}=0 \quad \text { and } \quad \omega_{n, 2} b_{1}+b_{2}\left(v_{r}^{2}+v_{i}^{2}\right)=0 . \quad(A=0) \text {, }
\end{aligned}
$$

which are equivalent to (6.4) and (6.7). Note that $\omega_{n, 2}$ can be of either sign which can affect the existence of these fixed points as $u_{r}^{2}+u_{i}^{2}$ and $v_{r}^{2}+v_{i}^{2}$ are both strictly positive. Let us consider the case $A \neq 0$ with $B=0$, with the corresponding result for $A=0$ and $B \neq 0$ being found similarly. Linearizing about the pure $A$ mode point (C 1 ), the equations for the perturbation quantities $\delta u_{r}, \delta u_{i}, \delta v_{r}$ and $\delta v_{i}$ satisfy

$$
\begin{aligned}
& \dot{\delta u_{r}}=\frac{2 a_{2}}{a_{0}} u_{r} u_{i} \delta u_{r}+\frac{2 a_{2}}{a_{0}} u_{i}^{2} \delta u_{i}, \\
& \dot{\delta u_{i}}=-\frac{2 a_{2}}{a_{0}} u_{r}^{2} \delta u_{r}-\frac{2 a_{2}}{a_{0}} u_{r} u_{i} \delta u_{i}, \\
& \dot{\delta v_{r}}=\frac{2 a_{4}}{b_{0}} u_{r} u_{i} \delta v_{r}+\frac{1}{b_{0}}\left[\omega_{n, 2} b_{1}+a_{3}\left(u_{r}^{2}+u_{i}^{2}\right)-a_{4}\left(u_{r}^{2}-u_{i}^{2}\right)\right] \delta v_{i}, \\
& \dot{\delta v_{i}}=-\frac{1}{b_{0}}\left[\omega_{n, 2} b_{1}+a_{3}\left(u_{r}^{2}+u_{i}^{2}\right)+a_{4}\left(u_{r}^{2}-u_{i}^{2}\right)\right] \delta v_{r}-\frac{2 a_{4}}{b_{0}} u_{r} u_{i} \delta v_{i} .
\end{aligned}
$$

The eigenvalues, $\lambda$, of this linear stability problem satisfy

$$
\operatorname{det}\left[\begin{array}{cccc}
\frac{2 a_{2}}{a_{0}} u_{r} u_{i}-\lambda & \frac{2 a_{2}}{a_{0}} u_{i}^{2} & 0 & 0 \\
-\frac{2 a_{2}}{a_{0}} u_{r}^{2} & -\frac{2 a_{2}}{a_{0}} u_{r} u_{i}-\lambda & 0 & 0 \\
0 & 0 & \frac{2 a_{4}}{b_{0}} u_{r} u_{i}-\lambda & c^{-} \\
0 & 0 & -c^{+} & -\frac{2 a_{4}}{b_{0}} u_{r} u_{i}-\lambda
\end{array}\right]=0,
$$

where

$$
c^{ \pm}=\frac{1}{b_{0}}\left[\omega_{n, 2} b_{1}+a_{3}\left(u_{r}^{2}+u_{i}^{2}\right) \pm a_{4}\left(u_{r}^{2}-u_{i}^{2}\right)\right] .
$$

The nontrivial eigenvalues then satisfy $(6.5)$ where $(\mathrm{C} 1)$ is used to eliminate the combination $u_{r}^{2}+u_{i}^{2}$.

Via a similar analysis, it can be shown that the nontrivial eigenvalues of the free $B$ modes satisfy (6.8).

\section{C.2. Stability of mixed modes $(A \neq 0$ and $B \neq 0)$}

To determine the stability at the fixed points for mixed modes, it is more convenient to write $(1.2)$ in polar form. Therefore, by writing

$$
A=r_{A} e^{\mathrm{i} \mu_{A}} \quad \text { and } \quad B=r_{B} e^{\mathrm{i} \mu_{B}},
$$

with $r_{A}>0$ and $r_{B}>0$, and the normal form equations (1.2) are transformed into the following four real equations for the amplitudes and phases

$$
\begin{aligned}
a_{0} \dot{r}_{A} & =a_{4} r_{A} r_{B}^{2} \sin \chi \\
b_{0} \dot{r}_{B} & =-a_{4} r_{B} r_{A}^{2} \sin \chi \\
-a_{0} r_{A} \dot{\mu}_{A} & =\left(\omega_{n, 2} a_{1}+a_{2} r_{A}^{2}+a_{3} r_{B}^{2}\right) r_{A}+a_{4} r_{A} r_{B}^{2} \cos \chi \\
-b_{0} r_{A} \dot{\mu}_{B} & =\left(\omega_{n, 2} b_{1}+b_{2} r_{B}^{2}+a_{3} r_{A}^{2}\right) r_{B}+a_{4} r_{B} r_{A}^{2} \cos \chi .
\end{aligned}
$$

Here $\chi=2\left(\mu_{B}-\mu_{A}\right)$ is the phase difference between the fixed points. In this case the fixed points satisfy

$$
\sin \chi=0 \quad \Longrightarrow \quad \cos \chi= \pm 1
$$


with the amplitudes satisfying the pair of algebraic equations (6.10) or (6.14). The solution for the general case is

$$
\begin{aligned}
r_{A}^{2} & =\frac{\omega_{n, 2}}{a_{2} b_{2}-\left(a_{3}+a_{4} \cos \chi\right)^{2}}\left[b_{2} a_{1}-b_{1}\left(a_{3}+a_{4} \cos \chi\right)\right], \\
r_{B}^{2} & =\frac{\omega_{n, 2}}{a_{2} b_{2}-\left(a_{3}+a_{4} \cos \chi\right)^{2}}\left[a_{2} b_{1}-a_{1}\left(a_{3}+a_{4} \cos \chi\right)\right] .
\end{aligned}
$$

For the mixed mode fixed points to exist, then $r_{A}^{2}$ and $r_{B}^{2}$ both have to be positive for the given values of $a_{i}, b_{i}$ and $\omega_{n, 2}$.

Assuming the fixed points exist, we determine their stability by linearising about these points. The linear evolution of the amplitudes and the phase difference are given by

$$
\begin{aligned}
\dot{\delta r_{A}} & =\frac{a_{4}}{a_{0}} r_{A} r_{B}^{2} \cos \chi \delta \chi \\
\dot{\delta r_{B}} & =-\frac{a_{4}}{b_{0}} r_{B} r_{A}^{2} \cos \chi \delta \chi \\
\dot{\delta \chi} & =4\left(\frac{a_{2}}{a_{0}}-\frac{a_{3}+a_{4} \cos \chi}{b_{0}}\right) r_{A} \delta r_{A}+4\left(\frac{a_{3}+a_{4} \cos \chi}{a_{0}}-\frac{b_{2}}{b_{0}}\right) r_{B} \delta r_{B},
\end{aligned}
$$

and the linear stability eigenvalues $\lambda$ satisfy

$$
\operatorname{det}\left[\begin{array}{ccc}
-\lambda & 0 & \frac{a_{4}}{a_{0}} r_{A} r_{B}^{2} \cos \chi \\
0 & -\lambda & -\frac{a_{4}}{b_{0}} r_{B} r_{A}^{2} \cos \chi \\
4\left(\frac{a_{2}}{a_{0}}-\frac{a_{3}+a_{4} \cos \chi}{b_{0}}\right) r_{A} & 4\left(\frac{a_{3}+a_{4} \cos \chi}{a_{0}}-\frac{b_{2}}{b_{0}}\right) r_{B} & -\lambda
\end{array}\right]=0 .
$$

Therefore, the nontrivial eigenvalues satisfy (6.12) for $\cos \chi=1$ and (6.16) for $\cos \chi=-1$.

\section{Appendix D. Equilibria in $\left(I_{1}, I_{2}, I_{3}\right)$ coordinates}

In the coordinate system on the spheroid, the pure mode with $A \neq 0$ and $B=0$ occurs at

$$
I_{1}=I_{2}=0 \text { and } I_{3}=a_{0} r_{A}^{2}>0 .
$$

The pure mode with $B \neq 0$ and $A=0$ occurs at

$$
I_{1}=I_{2}=0 \quad \text { and } \quad I_{3}=-b_{0} r_{B}^{2}<0 .
$$

For mixed modes the system can be written as

$$
\begin{aligned}
& I_{1}=2 r_{A} r_{B} \cos \left(\mu_{A}-\mu_{B}\right) \\
& I_{2}=2 r_{A} r_{B} \sin \left(\mu_{A}-\mu_{B}\right) \\
& I_{3}=a_{0} r_{A}^{2}-b_{0} r_{B}^{2} .
\end{aligned}
$$

Therefore, mixed modes with $\mu_{B}=\mu_{A}$ or $\mu_{B}=\mu_{A}+\pi$ occur at

$$
I_{1}= \pm 2 r_{A} r_{B}, \quad I_{2}=0, \quad I_{3}=a_{0} r_{A}^{2}-b_{0} r_{B}^{2}
$$

and mixed modes with $\mu_{B}=\mu_{A}+\frac{1}{2} \pi$ or $\mu_{B}=\mu_{A}+\frac{3}{2} \pi$ occur at

$$
I_{1}=0, \quad I_{2}= \pm 2 r_{A} r_{B}, \quad I_{3}=a_{0} r_{A}^{2}-b_{0} r_{B}^{2}
$$

\section{REFERENCES}

Adee, B. H. \& Caglayan, I. 1982 The effects of free water on deck on the motions and stability of vessels. In Proc. Second Inter. Conf. Stab. Ships and Ocean Vehicles. SNAME, Tokyo: Springer.

Alemi Ardakani, H. \& Bridges, T. J. 2010 Dynamic coupling between shallow-water sloshing and horizontal vehicle motion. Europ. J. Appl. Math 21, 479-517.

Alemi Ardakani, H., Bridges, T. J. \& Turner, M. R. $2012 a$ Resonance in a model for Cooker's sloshing experiment. Euro. J. Mech. B/Fluids 36, 25-38.

Alemi Ardakani, H., Bridges, T. J. \& Turner, M. R. $2012 b$ Resonance in a model for Cooker's sloshing experiment - extended version. Technical Report, University of Surrey http://personal.maths. surrey.ac.uk/st/T.Bridges/SLOSH/RESONANCE.

Caglayan, I. \& Storch, R. L. 1982 Stability of fishing vessels with water on deck: a review. J. Ship Research 26, 106-116. 
Cooker, M. J. 1994 Water waves in a suspended container. Wave Motion 20, 385-395.

Cotter, C. S. 1986 The 1:1 resonance and the Hénon-Heiles family of Hamiltonians. PhD Thesis, University of California, Santa Cruz, USA.

Cushman, R. \& Rod, D. L. 1982 Reduction of the semisimple $1: 1$ resonance. Physica D 6, 105-112.

Dillingham, J. 1981 Motion studies of a vessel with water on deck. Wave Motion 18, 38-50.

Faltinsen, O. M., Rognebakke, O. F. \& Timokha, A. N. 2003 Resonant three-dimensional nonlinear sloshing in a square-base basin. J. Fluid Mech. 487, 1-42.

Faltinsen, O. M. \& Timokha, A. N. 2009 Sloshing. Cambridge University Press.

Feng, Z. C. 1998 Coupling between neighboring two-dimensional modes of water waves. Phys. Fluids 10 (9), $2405-2411$.

Feng, Z. C. \& Sethna, P. R. 1989 Symmetry breaking bifurcations in resonant surface waves. J. Fluid Mech. 199, 495-518.

Frandsen, J. B. 2005 Numerical predictions of tuned liquid tank structural systems. J. Fluids $E$ S Structures 20 , 309-329.

Graham, E. W. \& Rodriguez, A. M. 1952 The characteristics of fuel motion which affect airplane dynamics. J. Appl. Mech. 19, 381-388.

HerczyŃski, A. \& Weidman, P. D. 2012 Experiments on the periodic oscillation of free containers driven by liquid sloshing. J. Fluid Mech. 693, 216-242.

Holmes, P. J. \& Marsden, J. E. 1983 Horseshoes and Arnold diffusion for Hamiltonian systems on Lie groups. Indiana U. Math. J. 32, 273-309.

Ibrahim, R. A. 2005 Liquid Sloshing Dynamics. Cambridge University Press.

Ikeda, T., Ibrahim, R. A., Harata, Y. \& Kuriyama, T. 2012 Nonlinear liquid sloshing in a square tank subjected to obliquely horizontal excitation. J. Fluid Mech. 700, 304-328.

IKEDA, T. \& NAKAGAWA, N. 1997 Non-linear vibrations of a structure caused by water sloshing in a rectangular tank. J. Sound Vibr. 201, 23-41.

Linton, C. M. \& McIver, P. 2001 Handbook of Mathematical Techniques for Wave-Structure Interaction. Chapman \& Hall/CRC: Boca Raton .

Luke, J. C. 1967 A variational principle for a fluid with a free surface. J. Fluid Mech. 27, 395-397.

Mayer, H. C. \& Krechetnikov, R. 2012 Walking with coffee: why does it spill? Phys. Rev. E 85, 046117.

Moiseyev, N. N. \& Rumyantsev, V. V. 1968 Dynamic Stability of Bodies Containing Fluid. Springer-Verlag.

Struble, R. A. \& Heinbockel, J. H. 1963 Resonant oscillations of a beam-pendulum system. J. Appl. Math. 30, 181-188.

Tadjbakhsh, I. \& Keller, J. B. 1960 Standing surface waves of finite amplitude. J. Fluid Mech. 8, $442-451$.

TAYloR, G. I. 1974 The interaction between experiment and theory in fluid mechanics. Ann. Rev. Fluid Mech. 6, $1-16$.

YU, J. 2010 Effects of finite water depth on natural frequencies of suspended water tanks. Stud. Appl. Math. 125, 337-391. 